\title{
GEOMETRIC STRUCTURE IN SMOOTH DUAL AND LOCAL LANGLANDS CONJECTURE
}

\author{
ANNE-MARIE AUBERT, PAUL BAUM, ROGER PLYMEN, AND MAARTEN SOLLEVELD
}

\section{CONTEnts}

1. Introduction

2. $\quad$-adic Fields

3. The Weil Group

4. Local Class Field Theory

5. Reductive $p$-adic Groups

6. The Smooth Dual

7. Split Reductive $p$-adic Groups

8. The Local Langlands Conjecture

9. The Hecke Algebra - Bernstein Components

10. Cuspidal Support Map - Tempered Dual 14

11. Extended Quotient $\quad 15$

12. Approximate Statement of the ABPS conjecture 16

13. Extended quotient of the second kind 16

14. Comparison of the two extended quotients 18

15. Statement of the ABPS conjecture 19

16. Two Theorems 23

17. Appendix : Geometric Equivalence 25

17.1. $k$-algebras 26

17.2. Spectrum preserving morphisms of $k$-algebras $\quad 27$

17.3. Algebraic variation of $k$-structure 28

17.4. Definition and examples 28

$\begin{array}{ll}\text { References } & 30\end{array}$

\section{InTRODUCTION}

The subject of this article is related to several branches of mathematics: number theory, representation theory, algebraic geometry, and non-commutative geometry. We start with a review of basic notions of number theory: $p$-adic field, the Weil group of a $p$-adic field, and local class field theory (sections 2-4). In sections 5, 6,7 , we recall some features of the theory of algebraic groups over $p$-adic fields and of their representations. In particular, we introduce the smooth dual $\operatorname{Irr}(G)$ of a reductive group $G$ over a $p$-adic field $F$. The smooth dual $\operatorname{Irr}(G)$ is the set of equivalence classes of irreducible smooth representations of $G$, and is the main

Paul Baum was partially supported by NSF grant DMS-0701184. 
object of study of this article. A detailed description of $\operatorname{Irr}(G)$, based on the Bernstein decomposition, is given in sections 9 and 10.

The Local Langlands Conjecture (the statement of which is the object of section 8) provides a markedly different way to understand $\operatorname{Irr}(G)$, by considering data that involve the Weil group of $F$ and a reductive complex group known as the Langlands dual group of $G$.

Starting with section 11, we introduce the extended quotient. This originated in non-commutative geometry. We use the extended quotient to state a conjecture, the ABPS conjecture, which asserts that a very simple geometric structure is present in $\operatorname{Irr}(G)$. We describe two cases in which the conjecture is known to be valid from our previous work. One of the features of the ABPS conjecture is that it provides a guide to determining $\operatorname{Irr}(G)$. A second feature is that it connects very closely to the local Langlands conjecture.

This expository article is based on the Takagi lectures given by the second author in November, 2012.

Topics in the lectures:

\#1. Review of the LL (Local Langlands) conjecture.

\#2. Statement of the ABPS (Aubert-Baum-Plymen-Solleveld) conjecture.

\#3. Brief indication of the proof that for any connected split reductive $p$-adic group $G$ both the ABPS and the LL conjectures are valid throughout the principal series of $G$.

Class field theory, a subject to which Professor Teiji Takagi made important and fundamental contributions, is a basic point in all three topics.

\section{P-ADIC FIELDS}

Let $K$ be a field, and $n$ a positive integer. $K^{\times}$is the multiplicative group of all non-zero elements of $K$.

$$
K^{\times}:=K-\{0\}
$$

$\mathrm{M}(n, K)$ is the $K$ vector space of all $n \times n$ matrices with entries in $K$.

$\mathrm{GL}(n, K)$ is the group of all $n \times n$ invertible matrices with entries in $K$.

$$
\operatorname{GL}(n, K):=\left\{\left[a_{i j}\right] \mid 1 \leq i, j \leq n \text { and } a_{i j} \in K \text { and } \operatorname{det}\left[a_{i j}\right] \neq 0\right\} .
$$

$\mathrm{SL}(n, K) \subset \mathrm{GL}(n, K)$ is the subgroup of $\mathrm{GL}(n, K)$ consisting of all $\left[a_{i j}\right] \in \mathrm{GL}(n, K)$ with $\operatorname{det}\left[a_{i j}\right]=1$.

$\mathrm{SO}(n, K)$ is the subgroup of $\mathrm{SL}(n, K)$ consisting of all $\left[a_{i j}\right] \in \mathrm{SL}(n, K)$ such that ${ }^{t}\left[a_{i j}\right]=\left[a_{i j}\right]^{-1}$, where ${ }^{t}\left[a_{i j}\right]$ is the transpose of $\left[a_{i j}\right]$.

$\operatorname{Sp}(2 n, K)$ is the subgroup of $\operatorname{SL}(2 n, K)$ consisting of all $\left[a_{i j}\right] \in \mathrm{SL}(2 n, K)$ with ${ }^{t}\left[a_{i j}\right] J\left[a_{i j}\right]=J$ where $J$ is the $2 n \times 2 n$ matrix

$$
J=\left[\begin{array}{cc}
0 & I_{n} \\
-I_{n} & 0
\end{array}\right]
$$


$I_{n}$ is the $n \times n$ identity matrix

$$
I_{n}=\left[\begin{array}{cccc}
1 & 0 & \ldots & 0 \\
0 & 1 & \ldots & 0 \\
\vdots & \vdots & \ddots & \vdots \\
0 & 0 & \ldots & 1
\end{array}\right]
$$

$K^{\times}$injects into $\operatorname{GL}(n, K)$ via $\lambda \mapsto \lambda I_{n}$, and $\operatorname{PGL}(n, K):=\operatorname{GL}(n, K) / K^{\times}$.

Fix a prime $p . p \in\{2,3,5,7,11,13,17, \ldots\}$. $\mathbb{Q}_{p}$ denotes the field of $p$-adic numbers. To construct $\mathbb{Q}_{p}$, for $n \neq 0, n \in \mathbb{Z}$, let $\operatorname{ord}_{p}(n)$ to be the largest $r \in\{0,1,2,3, \ldots\}$ such that $p^{r}$ divides $\mathrm{n}$.

$$
\operatorname{ord}_{p}(n):=\text { largest } r \in\{0,1,2,3, \ldots\} \text { such that } n \equiv 0\left(p^{r}\right)
$$

For $\frac{n}{m} \in \mathbb{Q}$, define $\left\|\frac{n}{m}\right\|_{p}$ by

$$
\left\|\frac{n}{m}\right\|_{p}:= \begin{cases}p^{\operatorname{ord}_{p}(m)-\operatorname{ord}_{p}(n)} & \text { if } \frac{n}{m} \neq 0 \\ 0 & \text { if } \frac{n}{m}=0\end{cases}
$$

For $x, y \in \mathbb{Q}$, set $\delta_{p}(x, y)=\|(x-y)\|_{p}$.

$$
\delta_{p}(x, y)=\|(x-y)\|_{p} \quad x, y \in \mathbb{Q}
$$

Then $\delta_{p}$ is a metric on $\mathbb{Q}$ and $\mathbb{Q}_{p}$ is the completion of $\mathbb{Q}$ using the metric $\delta_{p} \cdot \mathbb{Q}_{p}$ is a locally compact totally disconnected topological field. $\mathbb{Q}$ (topologized by the metric $\delta_{p}$ ) is a dense subfield.

$$
\mathbb{Q} \subset \mathbb{Q}_{p}
$$

Any nonzero element $x \in \mathbb{Q}_{p}$ is uniquely of the form :

$$
x=a_{n} p^{n}+a_{n+1} p^{n+1}+a_{n+2} p^{n+2}+\cdots
$$

where

$$
n \in \mathbb{Z}, \quad a_{j} \in\{0,1,2, \ldots, p-1\} \quad \text { and } \quad a_{n} \neq 0 .
$$

For example

$$
-1=(p-1) p^{0}+(p-1) p^{1}+(p-1) p^{2}+(p-1) p^{3}+\cdots
$$

Note that for any element $x \in \mathbb{Q}_{p}$, the "pole" $\sum_{r \leq-1} a_{r} p^{r}$ has at most finitely many non-zero terms.

For $x \in \mathbb{Q}_{p}^{\times}$with $x=a_{n} p^{n}+a_{n+1} p^{n+1}+a_{n+2} p^{n+2}+\cdots$, the valuation of $x$, denoted val $_{\mathbb{Q}_{p}}(x)$, is the smallest $j \in \mathbb{Z}$ with $a_{j} \neq 0$.

$$
\text { val }_{\mathbb{Q}_{p}}: \mathbb{Q}_{p}^{\times} \longrightarrow \mathbb{Z}
$$

is a homomorphism of topological groups where $\mathbb{Z}$ has the discrete topology and $\mathbb{Q}_{p}^{\times}$is topologized as a subspace of $\mathbb{Q}_{p}$.

Set

$$
\mathbb{Z}_{p}:=\left\{x \in \mathbb{Q}_{p}^{\times} \mid \operatorname{val}_{\mathbb{Q}_{p}}(x) \geq 0\right\} \cup\{0\} .
$$

Then:

- $\mathbb{Z}_{p}$ is a compact subring of $\mathbb{Q}_{p}$ - and is the unique maximal compact subring of $\mathbb{Q}_{p}$. 
- As a topological ring $\mathbb{Z}_{p}$ is isomorphic to the $p$-adic completion of $\mathbb{Z}$.

$$
\mathbb{Z}_{p} \cong \lim _{\infty \leftarrow n}\left(\mathbb{Z} / p^{n} \mathbb{Z}\right)
$$

- $\mathbb{Z}_{p}$ is a local ring whose unique maximal ideal $\mathcal{J}_{p}$ is :

$$
\mathcal{J}_{p}=\left\{x \in \mathbb{Q}_{p}^{\times} \mid \operatorname{val}(x) \geq 1\right\} \cup\{0\}
$$

- The quotient $\mathbb{Z}_{p} / \mathcal{J}_{p}$ is the finite field with $p$ elements.

$$
\mathbb{Z}_{p} / \mathcal{J}_{p} \cong \mathbb{Z} / p \mathbb{Z}
$$

$\mathbb{Z}_{p}$ is the integers of $\mathbb{Q}_{p}$ and $\mathbb{Z}_{p} / \mathcal{J}_{p}$ is the residue field of $\mathbb{Q}_{p}$.

Definition. A p-adic field is a field $F$ which is a finite extension of $\mathbb{Q}_{p}$ :

$$
F \supset \mathbb{Q}_{p} \text { and } \operatorname{dim}_{\mathbb{Q}_{p}} F<\infty
$$

Any $p$-adic field $F$ is a locally compact totally disconnected topological field.

The valuation val $_{\mathbb{Q}_{p}}: \mathbb{Q}_{p}^{\times} \rightarrow \mathbb{Z}$ of $\mathbb{Q}_{p}$ extends to give the non-normalized valuation

$$
F^{\times} \longrightarrow \mathbb{Q} \text {. }
$$

This is a group homomorphism from $F^{\times}$to the additive group of rational numbers. The image of this homomorphism has a smallest positive element $s$. Dividing by $s$ then gives the normalized valuation of $F$,

$$
\operatorname{val}_{F}: F^{\times} \longrightarrow \mathbb{Z} .
$$

$v a l_{F}$ is a surjective homomorphism of topological groups where $\mathbb{Z}$ has the discrete topology and $F^{\times}$is topologized as a subspace of $F$.

The integers of $F$, denoted $\mathcal{Z}_{F}$, is defined by :

$$
\mathcal{Z}_{F}:=\left\{x \in F^{\times} \mid \operatorname{val}_{F}(x) \geq 0\right\} \cup\{0\} .
$$

Then:

- $\mathcal{Z}_{F}$ is a compact subring of $F$ - and is the unique maximal compact subring of $F$.

- $\mathcal{Z}_{F}$ is a local ring whose unique maximal ideal $\mathcal{J}_{F}$ is:

$$
\mathcal{J}_{F}=\left\{x \in F^{\times} \mid \operatorname{val}_{F}(x) \geq 1\right\} \cup\{0\} .
$$

- The quotient $\mathcal{Z}_{F} / \mathcal{J}_{F}$ is a finite field which is an extension of the field with $p$ elements:

$$
\mathcal{Z}_{F} / \mathcal{J}_{F} \supset \mathbb{Z} / p \mathbb{Z}
$$

The field $\mathcal{Z}_{F} / \mathcal{J}_{F}$ is called the residue field of $F$. 


\section{The Weil Group}

Let $F$ be a $p$-adic field. $\bar{F}$ denotes the algebraic closure of $F . \operatorname{Gal}(\bar{F} \mid F)$ is the Galois group of $\bar{F}$ over $F$, i.e. $\operatorname{Gal}(\bar{F} \mid F)$ is the group consisting of all automorphisms $\varphi$ of the field $\bar{F}$ such that

$$
\varphi(x)=x \quad \forall x \in F
$$

With its natural topology $\operatorname{Gal}(\bar{F} \mid F)$ is a compact totally disconnected topological group and comes equipped with a continuous surjection onto the pro-finite completion of $\mathbb{Z}$.

$$
\operatorname{Gal}(\bar{F} \mid F) \rightarrow \widehat{\mathbb{Z}}:=\lim _{\infty \leftarrow n}(\mathbb{Z} / n \mathbb{Z})
$$

The group $\widehat{\mathbb{Z}}$ is isomorphic to the absolute Galois group of the residue field $\mathcal{Z}_{F} / \mathcal{J}_{F}$. It has a canonical generator, called a geometric Frobenius element. The kernel of the continuous surjection $\operatorname{Gal}(\bar{F} \mid F) \rightarrow \widehat{\mathbb{Z}}$ is denoted $\mathcal{I}_{F}$ and is the inertia group of $F$. Consider the short exact sequence of locally compact totally disconnected topological groups

$$
1 \longrightarrow \mathcal{I}_{F} \hookrightarrow \operatorname{Gal}(\bar{F} \mid F) \rightarrow \widehat{\mathbb{Z}} \longrightarrow 0 .
$$

$\mathbb{Z}$ injects into $\widehat{\mathbb{Z}}$ via the inclusion $\mathbb{Z} \subset \widehat{\mathbb{Z}}$. Within $\operatorname{Gal}(\bar{F} \mid F)$ let $\mathcal{W}_{F}^{\diamond}$ be the pre-image of $\mathbb{Z}$ so that there is the short exact sequence of topological groups

$$
1 \longrightarrow \mathcal{I}_{F} \hookrightarrow \mathcal{W}_{F}^{\diamond} \rightarrow \mathbb{Z} \longrightarrow 0 .
$$

Note that in this short exact sequence $\mathcal{I}_{F}$ and $\mathcal{W}_{F}^{\diamond}$ are topologized as subspaces of $\operatorname{Gal}(\bar{F} \mid F) . \mathbb{Z}$ is topologized as a subspace of $\widehat{\mathbb{Z}}$, so in this short exact sequence $\mathbb{Z}$ does not have the discrete topology.

Minimally enlarge the collection of open sets in $\mathcal{W}_{F}^{\diamond}$ so that with this new topology the map $\mathcal{W}_{F}^{\diamond} \rightarrow \mathbb{Z}$ is continuous where $\mathbb{Z}$ now has the discrete topology. $\mathcal{W}_{F}^{\diamond}$ with this new topology is the Weil group of $F[14]$ and is denoted $\mathcal{W}_{F} . \mathcal{W}_{F}$ is a topological group and there is the short exact sequence of topological groups

$$
1 \longrightarrow \mathcal{I}_{F} \hookrightarrow \mathcal{W}_{F} \rightarrow \mathbb{Z} \longrightarrow 0,
$$

in which $\mathbb{Z}$ has the discrete topology. $\mathcal{I}_{F}$ is an open (and closed) subgroup of $\mathcal{W}_{F}$. The topology that $\mathcal{I}_{F}$ receives as a subspace of $\mathcal{W}_{F}$ is the same as its topology as a subspace of $\operatorname{Gal}(\bar{F} \mid F)$.

Denote the continuous map $\mathcal{W}_{F} \rightarrow \mathbb{Z}$ by $\varepsilon: \mathcal{W}_{F} \rightarrow \mathbb{Z}$.

\section{Local Class Field Theory}

As above, $F$ is a $p$-adic field and $\mathcal{W}_{F}$ is the Weil group of $F . \mathcal{W}_{F}^{\text {der }}$ is the derived group of $\mathcal{W}_{F}$, i.e. $\mathcal{W}_{F}^{\text {der }}$ is the closure (in $\mathcal{W}_{F}$ ) of the commutator subgroup. The abelianization of $\mathcal{W}_{F}$, denoted $\mathcal{W}_{F}^{\mathrm{ab}}$, is the topological group which is the quotient $\mathcal{W}_{F} / \mathcal{W}_{F}^{\text {der }}$ with the quotient topology.

$$
\mathcal{W}_{F}^{\mathrm{ab}}:=\mathcal{W}_{F} / \mathcal{W}_{F}^{\mathrm{der}}
$$

Local class field theory [20] asserts that there is a canonically defined surjective homomorphism of topological groups

$$
\alpha_{F}: \mathcal{W}_{F} \longrightarrow F^{\times}
$$


with commutativity in the diagram

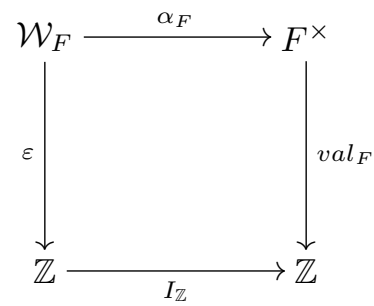

$\left(I_{\mathbb{Z}}=\right.$ the identity map of $\left.\mathbb{Z}\right)$ such that the map $\alpha_{F}$ induces an isomorphism of topological groups.

$$
\alpha_{F}: \mathcal{W}_{F}^{\mathrm{ab}} \cong F^{\times}
$$

$\alpha_{F}$ is called the Artin reciprocity map.

\section{Reductive $p$-ADIC Groups}

If $k$ is any field and $V$ is a finite dimensional $k$ vector space, there are the polynomial functions $V \rightarrow k$. If $k^{\prime}$ is an extension of $k$, then any polynomial function $V \rightarrow k$ extends canonically to give a polynomial function $k^{\prime} \otimes_{k} V \rightarrow k^{\prime}$.

As above, $F$ is a p-adic field and $\bar{F}$ is the algebraic closure of $F$.

A subgroup $G \subset \mathrm{GL}(n, F)$ is algebraic if there exist polynomial functions $P_{1}, P_{2}, \ldots, P_{r}$

$$
P_{j}: \mathrm{M}(n, F) \longrightarrow F \quad j=1,2, \ldots, r
$$

such that

(1) $G=\left\{g \in \mathrm{GL}(n, F) \mid P_{j}(g)=0 \quad j=1,2, \ldots, r\right\}$

(2) $\bar{G}:=\left\{g \in \operatorname{GL}(n, \bar{F}) \mid P_{j}(g)=0 \quad j=1,2, \ldots, r\right\}$ is a $\operatorname{subgroup~of~} \operatorname{GL}(n, \bar{F})$.

An algebraic group $G \subset \operatorname{GL}(n, F)$ is topologized by the topology it receives from $F$. In this topology $G$ is a locally compact and totally disconnected topological group. $\bar{G}$, however, is topologized in a quite different way. $\bar{G}$ is an affine variety over the algebraically closed field $\bar{F}$. So $\bar{G}$ is topologized by the Zariski topology in which the closed sets are the algebraic sub-varieties of $\bar{G}$.

The algebraic group $G$ is called connected if $\bar{G}$ is connected in the Zariski topology. We note that this only means connectedness as an algebraic variety, it does not refer to $G$ as a space with the locally compact topology from $F$.

$g=\left[a_{i j}\right] \in \bar{G}$ is unipotent if all the eigenvalues of $g$ are 1 . A subgroup $H \subset \bar{G}$ is unipotent if every $g \in H$ is unipotent. An algebraic group $G \subset \operatorname{GL}(n, F)$ is a reductive $p$-adic group if the only connected normal unipotent subgroup of $\bar{G}$ is the trivial one-element subgroup.

Examples. The groups $\mathrm{GL}(n, F), \mathrm{SL}(n, F), \mathrm{PGL}(n, F), \mathrm{SO}(n, F), \mathrm{Sp}(2 n, F)$ are connected reductive $p$-adic groups.

Example. With $n \geq 2$, let $U T(n, F)$ be the subgroup of $\operatorname{GL}(n, F)$ consisting of all upper triangular matrices.

$$
U T(n, F):=\left\{\left[a_{i j}\right] \in \mathrm{GL}(n, F) \mid a_{i j}=0 \text { if } i>j\right\}
$$


In $U T(n, \bar{F})$ consider the subgroup consisting of those $\left[a_{i j}\right] \in U T(n, \bar{F})$ such that $a_{i j}=1$ if $i=j$. This is a non-trivial connected normal unipotent subgroup of $U T(n, \bar{F})$. So $U T(n, F)$ is not reductive.

\section{The Smooth Dual}

Let $G$ be a reductive $p$-adic group.

Definition. A representation of $G$ is a group homomorphism

$$
\phi: G \rightarrow \operatorname{Aut}_{\mathbb{C}}(V)
$$

where $V$ is a vector space over the complex numbers $\mathbb{C}$.

Definition. Two representations of $G$

$$
\phi: G \rightarrow \operatorname{Aut}_{\mathbb{C}}(V)
$$

and

$$
\psi: G \rightarrow \operatorname{Aut}_{\mathbb{C}}(W)
$$

are equivalent if there exists an isomorphism of $\mathbb{C}$ vector spaces $T: V \rightarrow W$ such that for all $g \in G$ there is commutativity in the diagram

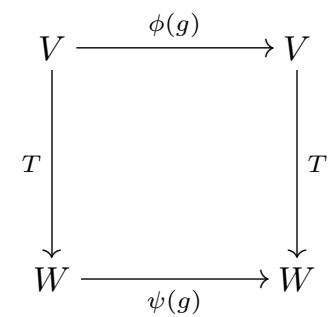

Definition. A representation

$$
\phi: G \rightarrow \operatorname{Aut}_{\mathbb{C}}(V)
$$

of $G$ is irreducible if $V \neq\{0\}$ and there is no vector subspace $W$ of $V$ such that $W$ is preserved by the action of $G,\{0\} \neq W$, and $W \neq V$.

Definition. A representation

$$
\phi: G \rightarrow \operatorname{Aut}_{\mathbb{C}}(V)
$$

of $G$ is smooth if for every $v \in V$ the stabilizer group

$$
G_{v}=\{g \in G \mid \phi(g) v=v\}
$$

is an open subgroup of $G$.

The smooth dual of $G$, denoted $\operatorname{Irr}(G)$, is the set of equivalence classes of smooth irreducible representations of $G$.

$$
\operatorname{Irr}(G)=\{\text { Smooth irreducible representations of } G\} / \sim
$$

Remark. In any topological group an open subgroup is also closed. This is due to the fact that if $H$ is an open subgroup of a topological group $G$, then any coset $g H$ is an open subset of $G \Longrightarrow G-\{H\}$ is open $\Longrightarrow H$ is closed.

Remark. In a reductive $p$-adic group $G$, if $U$ is any open set with the identity element $e$ an element of $U$, then there is a compact open subgroup $H$ with $H \subset U$. 


\section{Split Reductive $p$-Adic Groups}

Let $G \subset \mathrm{GL}(n, F)$ be a reductive $p$-adic group. If $r$ is a natural number, then $\left(\bar{F}^{\times}\right)^{r}$ is the Cartesian product of $r$ copies of $\bar{F}^{\times}$.

$$
\left(\bar{F}^{\times}\right)^{r}:=\bar{F}^{\times} \times \bar{F}^{\times} \times \cdots \times \bar{F}^{\times}
$$

A torus in $\bar{G}$ is a closed connected abelian subgroup $\mathcal{T}$ of $\bar{G}$ such that for some $r \in \mathbb{Z}_{\geq 0}$ there exists a bijection

$$
\psi: \mathcal{T} \longmapsto\left(\bar{F}^{\times}\right)^{r}
$$

with $\psi$ both a group homomorphism and an isomorphism of $\bar{F}$ affine varieties.

$G$ is split if there exists an algebraic subgroup $\mathbb{T}$ of $\mathrm{GL}(n, F)$ such that

(1) $\mathbb{T} \subset G$ and $\overline{\mathbb{T}} \subset \bar{G}$.

(2) $\overline{\mathbb{T}}$ is a maximal torus in $\bar{G}$.

(3) The bijection $\psi: \overline{\mathbb{T}} \longmapsto\left(\bar{F}^{\times}\right)^{r}$ can be chosen to be defined over $F$.

In (3) "defined over $F$ " means that the polynomials which give the bijection $\psi: \overline{\mathbb{T}} \longmapsto\left(\bar{F}^{\times}\right)^{r}$ have their coefficients in $F$. Note that in this case, when restricted to $\mathbb{T}, \psi$ gives an isomorphism of locally compact totally disconnected topological groups

$$
\mathbb{T} \longmapsto\left(F^{\times}\right)^{r} .
$$

If $G$ is a split reductive p-adic group, then a subgroup $\mathbb{T}$ of $G$ which satisfies conditions (1), (2), (3) is a maximal $p$-adic torus in $G$. Any two maximal $p$-adic tori in $G$ are conjugate.

Examples. The groups $\mathrm{GL}(n, F), \mathrm{SL}(n, F), \mathrm{PGL}(n, F), \mathrm{SO}(n, F), \mathrm{Sp}(2 n, F)$ are connected split reductive $p$-adic groups.

Notation. Let $G \subset \operatorname{GL}(n, F)$ be a reductive $p$-adic group. If $E$ is a finite extension of $F$, then $G_{E}$ is the intersection of $\bar{G}$ with $\operatorname{GL}(n, E)$.

$$
G_{E}:=\bar{G} \cap \operatorname{GL}(n, E)
$$

For any finite extension $E$ of $F, G_{E}$ is a reductive group over the $p$-adic field $E$, and $G$ split implies $G_{E}$ split.

Proposition. Let $G \subset \mathrm{GL}(n, F)$ be a reductive $p$-adic group. Then there is a finite extension $E$ of $F$ such that $G_{E}$ is split.

The proposition guarantees that the split reductive $p$-adic groups are plentiful within the reductive $p$-adic groups.

Let $G$ be a reductive $p$-adic group. A connected algebraic subgroup $\bar{B}$ of $\bar{G}$ is a Borel subgroup of $\bar{G}$ if $\bar{B}$ is solvable and $\bar{B}$ is maximal among the connected solvable algebraic subgroups of $\bar{G}$. $G$ is quasi-split if $\bar{G}$ has a Borel subgroup $\bar{B}$ defined over $F$ - i.e. the polynomials determining $\bar{B}$ can be chosen to have their coefficients in $F$, where $F$ is the $p$-adic field over which $G$ is defined. In this case $G$ itself has a Borel subgroup, namely

$$
B:=\bar{B} \cap G
$$

Any split group $G$ is quasi-split. 


\section{The Local Langlands Conjecture}

Let $G$ be a connected reductive $p$-adic group. Associated to $G$ is a connected reductive algebraic group over the complex numbers, called the dual group of $G$ and denoted here by ${ }^{L} G^{\circ}$.

Examples.

$$
\begin{array}{rr}
G=\mathrm{GL}(n, F) & { }^{L} G^{\circ}=\mathrm{GL}(n, \mathbb{C}) \\
G=\mathrm{SL}(n, F) & { }^{L} G^{\circ}=\operatorname{PGL}(n, \mathbb{C}) \\
G=\operatorname{PGL}(n, F) & { }^{L} G^{\circ}=\mathrm{SL}(n, \mathbb{C}) \\
G=\mathrm{SO}(2 n, F) & { }^{L} G^{\circ}=\mathrm{SO}(2 n, \mathbb{C}) \\
G=\mathrm{SO}(2 n+1, F) & { }^{L} G^{\circ}=\mathrm{Sp}(2 n, \mathbb{C}) \\
G=\mathrm{Sp}(2 n, F) & { }^{L} G^{\circ}=\mathrm{SO}(2 n+1, \mathbb{C})
\end{array}
$$

As above, $F$ is the $p$-adic field over which $G$ is defined. It is possible to make $\operatorname{Gal}(\bar{F} \mid F)$ act on ${ }^{L} G^{\circ}$ as automorphisms of the complex algebraic group ${ }^{L} G^{\circ}$. The L-group of $G$, denoted ${ }^{L} G$, which was introduced by Langlands in his original paper [24], is the semidirect product ${ }^{L} G^{\circ} \rtimes \operatorname{Gal}(\bar{F} \mid F)$,

$$
{ }^{L} G:={ }^{L} G^{\circ} \rtimes \operatorname{Gal}(\bar{F} \mid F) .
$$

Via the usual topologies for ${ }^{L} G^{\circ}$ and $\operatorname{Gal}(\bar{F} \mid F),{ }^{L} G$ is a locally compact Hausdorff topological group. Since ${ }^{L} G^{\circ}$ is connected and $\operatorname{Gal}(\bar{F} \mid F)$ is totally disconnected, ${ }^{L} G^{\circ}$ is the connected component of the identity in ${ }^{L} G$.

A torus in ${ }^{L} G^{\circ}$ is an algebraic subgroup $T$ of ${ }^{L} G^{\circ}$ such that for some $r \in \mathbb{Z}_{\geq 0}$ there exists a bijection

$$
\psi: T \longmapsto\left(\mathbb{C}^{\times}\right)^{r}=\mathbb{C}^{\times} \times \mathbb{C}^{\times} \times \cdots \times \mathbb{C}^{\times},
$$

with $\psi$ both a group homomorphism and an isomorphism of complex affine varieties. Any two maximal tori in ${ }^{L} G^{\circ}$ are conjugate. $g \in{ }^{L} G^{\circ}$ is semi-simple if there is a torus $T$ in ${ }^{L} G^{\circ}$ with $g \in T$.

More generally, $\beta \in{ }^{L} G$ is said to be semi-simple if, whenever $\pi:{ }^{L} G \rightarrow \operatorname{Aut}_{\mathbb{C}} V$ is a finite dimensional representation of ${ }^{L} G, \pi(\beta)$ is semi-simple.

Notation. $\left({ }^{L} G\right)_{\text {semi-simple }}$ denotes the set of all semi-simple elements in ${ }^{L} G$.

A Langlands parameter for a connected reductive $p$-adic group $G$ is a group homomorphism

$$
\varphi: \mathcal{W}_{F} \times \mathrm{SL}(2, \mathbb{C}) \longrightarrow{ }^{L} G
$$

such that

- $\varphi$ is continuous.

- Restricted to $\mathrm{SL}(2, \mathbb{C}), \varphi$ is a morphism of complex algebraic groups $\operatorname{SL}(2, \mathbb{C}) \rightarrow{ }^{L} G^{\circ}$. The complex dual group ${ }^{L} G^{\circ}$ is the connected component of the identity in ${ }^{L} G$. Since $\varphi$ is continuous and $\operatorname{SL}(2, \mathbb{C})$ is connected, $\varphi$ must map $\operatorname{SL}(2, \mathbb{C})$ to ${ }^{L} G^{\circ}$. 
- There is commutativity in the diagram

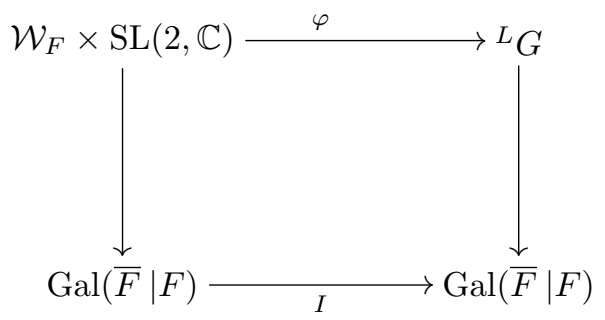

In this diagram $I: \operatorname{Gal}(\bar{F} \mid F) \rightarrow \operatorname{Gal}(\bar{F} \mid F)$ is the identity map $I(\beta)=\beta \quad \forall \beta \in \operatorname{Gal}(\bar{F} \mid F)$. The right vertical arrow is the quotient map obtained by dividing ${ }^{L} G={ }^{L} G^{\circ} \rtimes \operatorname{Gal}(\bar{F} \mid F)$ by the normal subgroup ${ }^{L} G^{\circ}$. The left vertical arrow is the composition $\mathcal{W}_{F} \times \mathrm{SL}(2, \mathbb{C}) \rightarrow \mathcal{W}_{F} \hookrightarrow \operatorname{Gal}(\bar{F} \mid F)$ where the first map is the evident projection and the second map is the evident inclusion.

- $\varphi\left(\mathcal{W}_{F}\right) \subset\left({ }^{L} G\right)_{\text {semi-simple }}$

The group ${ }^{L} G^{\circ}$ acts on the set of Langlands parameters for $G$ by conjugation, so one can form the quotient set

$$
\Phi(G):=\{\text { Langlands parameters for } G\} /{ }^{L} G^{\circ} .
$$

The local Langlands conjecture (LLC) $[24,15,14]$ asserts that there is a map of sets

$$
\alpha_{G}: \operatorname{Irr}(G) \longrightarrow \Phi(G)
$$

which is finite-to-one and has important properties. It is expected that $\alpha_{G}$ is surjective if $G$ is quasi-split. The fibers of $\alpha_{G}$ are referred to as L-packets.

The LLC was proved for $\operatorname{GL}(n, F)$ in Harris-Taylor[17] and Henniart[18], and subseqently reproved by Scholze[32]. Earlier results of Zelevinsky[35] are relevant. For $G=\mathrm{GL}(n, F)$, the map $\alpha_{G}$ is bijective, and uniqueness is secured via a wellknown set of matching conditions, including compatibility of the $L$-factors and $\epsilon$ factors for pairs involved in the local Rankin-Selberg convolutions. When combined with the Jacquet-Langlands correspondence, this proves the LLC for inner forms $\mathrm{GL}(m, D)$ of $\mathrm{GL}(m d, F)[19,7]$.

For recent LLC results, see Arthur [2] and Mok[29]. The groups considered in [2] are symplectic and orthogonal groups. Modulo stabilization of the twisted trace formula for general linear groups (which is work in progress of Waldspurger and others), Arthur [2] classifies the L-packets of tempered representations. The groups considered in [29] are quasi-split unitary groups. Using a method analogous to the work of Arthur[2], Mok [29] classifies the L-packets of tempered representations of quasi-split unitary groups.

In [9], a proof is given that results of Arthur-Mok type (i.e. classification of Lpackets of tempered representations) can be used to construct the local Langlands correspondence for the smooth dual $\operatorname{Irr}(G)$.

If $G$ is connected and split — or, more generally, if $G$ is an inner form of a connected split reductive $p$-adic group - then the action of $\operatorname{Gal}(\bar{F} \mid F)$ on ${ }^{L} G^{\circ}$ is 
trivial. Hence in this case, ${ }^{L} G$ is the product group ${ }^{L} G^{\circ} \times \operatorname{Gal}(\bar{F} \mid F)$.

$$
G \text { connected and split } \Longrightarrow{ }^{L} G={ }^{L} G{ }^{\circ} \times \operatorname{Gal}(\bar{F} \mid F)
$$

Notation. For the rest of this section $G$ will be a connected split reductive $p$-adic group or an inner form of such a group.

"inner form" = "has same Langlands dual group".

For such a $G$, the definition of Langlands parameter given above is equivalent to Langlands parameter defined as follows.

A Langlands parameter for a connected split reductive $p$-adic group $G$ is a group homomorphism

$$
\varphi: \mathcal{W}_{F} \times \mathrm{SL}(2, \mathbb{C}) \longrightarrow{ }^{L} G^{\circ}
$$

such that

(1) Restricted to $\mathrm{SL}(2, \mathbb{C}), \varphi$ is a morphism of complex algebraic groups.

(2) Restricted to $\mathcal{W}_{F}, \varphi$ is continuous where ${ }^{L} G^{\circ}$ is topologized by viewing it as the underlying locally compact Hausdorff space of the complex analytic manifold ${ }^{L} G^{\circ}$.

(3) $\varphi\left(\mathcal{W}_{F}\right) \subset{ }^{L} G^{\circ}$ semi-simple.

For (3) one uses that, since $G$ is split,

$$
\left({ }^{L} G\right)_{\text {semi-simple }}={ }^{L} G^{\circ}{ }_{\text {semi-simple }} \times \operatorname{Gal}(\bar{F} \mid F) .
$$

(2) implies that $\varphi\left(\mathcal{I}_{F}\right)$ is a finite subgroup of ${ }^{L} G^{\circ} \cdot \varphi\left(\mathcal{W}_{F}\right)$ is the subgroup of ${ }^{L} G^{\circ}$ generated by $\varphi\left(\mathcal{I}_{F}\right)$ and one additional semi-simple element of ${ }^{L} G^{\circ}$, the image of a geometric Frobenius element of $\mathcal{W}_{F} \cdot \varphi(\operatorname{SL}(2, \mathbb{C}))$ is either trivial (i.e. is the trivial one-element subgroup of ${ }^{L} G^{\circ}$ ) or is an algebraic subgroup of ${ }^{L} G^{\circ}$ isomorphic to $\operatorname{SL}(2, \mathbb{C})$ or $\operatorname{PSL}(2, \mathbb{C}):=\operatorname{SL}(2, \mathbb{C}) /\left\{I_{2},-I_{2}\right\}$.

The group ${ }^{L} G^{\circ}$ acts on the set of Langlands parameters for $G$ by conjugation, so again we can form the quotient set

$$
\Phi(G):=\{\text { Langlands parameters for } G\} /{ }^{L} G^{\circ} .
$$

As recalled above, the local Langlands conjecture (LLC) asserts that there is a map of sets

$$
\alpha_{G}: \operatorname{Irr}(G) \longrightarrow \Phi(G)
$$

which is surjective, finite-to-one and has important properties. The fibers of $\alpha_{G}$ are referred to as $L$-packets.

Example. Let $G=\operatorname{GL}(1, F)=F^{\times}$. Then ${ }^{L} G^{\circ}=\operatorname{GL}(1, \mathbb{C})=\mathbb{C}^{\times}$. Any morphism of complex algebraic groups $\operatorname{SL}(2, \mathbb{C}) \rightarrow \mathbb{C}^{\times}$is trivial, so in this example $\{$ Langlands parameters for $G\} /{ }^{L} G^{\circ}=\left\{\right.$ continuous homomorphisms $\left.\mathcal{W}_{F} \rightarrow \mathbb{C}^{\times}\right\}$. The isomorphism of local class field theory

$$
\mathcal{W}_{F}^{\mathrm{ab}} \cong F^{\times}
$$

gives a bijection

$$
\left\{\text { continuous homomorphisms } \mathcal{W}_{F} \rightarrow \mathbb{C}^{\times}\right\} \longleftrightarrow \widehat{F^{\times}}
$$

which verifies LLC for this example and produces the point of view that the goal of LLC is to extend local class field theory to non-abelian connected reductive $p$-adic groups. 
Let $D$ be a division algebra of dimension $d^{2}$ over its center $F$. With $m$ a positive integer, $\operatorname{GL}(m, D)$ denotes the connected reductive $p$-adic group consisting of all $m \times m$ invertible matrices with entries in $D$. Except for $\operatorname{GL}(n, F)$, the groups $\mathrm{GL}(m, D)$ are non-split. The group $\mathrm{GL}(m, D)$ is an inner form of $\mathrm{GL}(m d, F)$ - hence $\operatorname{GL}(m, D)$ and $\operatorname{GL}(m d, F)$ have the same Langlands dual group. Since $\mathrm{GL}(n, F)$ is connected and split, each group $\mathrm{GL}(m, D)$ is an inner form of a connected split reductive $p$-adic group.

Each group $G=\mathrm{GL}(m, D)$ has a reduced norm map

$$
\text { Nrd: GL }(m, D) \longrightarrow F^{\times} \text {. }
$$

Set $G^{\sharp}=\operatorname{ker}\left(\operatorname{GL}(m, D) \rightarrow F^{\times}\right)$. The group $G^{\sharp}$ is an inner form of $\operatorname{SL}(m d, F)$. LLC for $G^{\sharp}$ is implied by LLC for $G$ - in the sense that each $L$-packet of $G^{\sharp}$ consists of the irreducible constituents of $\operatorname{Res}_{G^{\sharp}}^{G}\left(\Pi_{\varphi}(G)\right)$ of an L-packet $\Pi_{\varphi}(G)$ of $G$ restricted to $G^{\sharp}$ - i.e. each L-packet of $G^{\sharp}$ consists of the irreducible constituents of the restriction to $G^{\sharp}$ of a smooth irreducible representation of $G$. A parametrization of the members of L-packets was found for the groups $G^{\sharp}$ in $[19,8]$.

\section{The Hecke Algebra - Bernstein Components}

Let $G$ be a reductive $p$-adic group. $G$ is locally compact so a left-invariant Haar measure $d g$ can be chosen. As every reductive group is unimodular, $d g$ is also rightinvariant. The Hecke algebra of $G$, denoted $\mathcal{H} G$, is then the convolution algebra of all locally-constant compactly-supported complex-valued functions $f: G \rightarrow \mathbb{C}$.

$$
\begin{aligned}
& (f+h)(g)=f(g)+h(g) \\
& (f * h)\left(g_{0}\right)=\int_{G} f(g) h\left(g^{-1} g_{0}\right) d g
\end{aligned}\left\{\begin{array}{l}
g \in G \\
g_{0} \in G \\
f \in \mathcal{H} G \\
h \in \mathcal{H} G
\end{array}\right.
$$

Definition. A representation of the Hecke algebra $\mathcal{H} G$ is a homomorphism of $\mathbb{C}$ algebras

$$
\psi: \mathcal{H} G \rightarrow \operatorname{End}_{\mathbb{C}}(V)
$$

where $V$ is a vector space over the complex numbers $\mathbb{C}$.

Definition. A representation

$$
\psi: \mathcal{H} G \rightarrow \operatorname{End}_{\mathbb{C}}(V)
$$

of the Hecke algebra $\mathcal{H} G$ is irreducible if $\psi: \mathcal{H} G \rightarrow \operatorname{End}_{\mathbb{C}}(V)$ is not the zero map and there exists no vector subspace $W$ of $V$ with $W$ preserved by the action of $\mathcal{H} G$ and $0 \neq W$ and $W \neq V$.

Definition. A primitive ideal in $\mathcal{H} G$ is an ideal $I$ which is the null space of an irreducible representation. Thus an ideal $I \subset \mathcal{H} G$ is primitive if and only if there exists an irreducible representation $\psi: \mathcal{H} G \rightarrow \operatorname{End}_{\mathbb{C}}(V)$ such that

$$
0 \longrightarrow I^{\complement} \mathcal{H} G \stackrel{\psi}{\longrightarrow} \operatorname{End}_{\mathbb{C}}(V)
$$

is exact. 
Remark. The Hecke algebra $\mathcal{H} G$ does not have a unit (unless $G=1$ ). $\mathcal{H} G$ does, however, have local units - i.e. if $a_{1}, a_{2}, a_{3}, \ldots, a_{l}$ is any finite set of elements of $\mathcal{H} G$, then there is an idempotent $\omega \in \mathcal{H} G$ with

$$
\omega a_{j}=a_{j} \omega=a_{j} \quad j=1,2, \ldots, l .
$$

Definition. A representation

$$
\psi: \mathcal{H} G \rightarrow \operatorname{End}_{\mathbb{C}}(V)
$$

of the Hecke algebra $\mathcal{H} G$ is non-degenerate if $(\mathcal{H} G) V=V$ - i.e. for each $v \in V$ there are $v_{1}, v_{2}, \ldots, v_{r} \in V$ and $f_{1}, f_{2}, \ldots, f_{r} \in \mathcal{H} G$ with $v=f_{1} v_{1}+f_{2} v_{2}+\cdots+f_{r} v_{r}$.

Remark. Any irreducible representation of $\mathcal{H} G$ is non-degenerate.

Let

$$
\phi: G \rightarrow \operatorname{Aut}_{\mathbb{C}}(V)
$$

be a smooth representation of $G$. Then $\phi$ integrates to give a non-degenerate representation of $\mathcal{H} G$ :

$$
f \mapsto \int_{G} f(g) \phi(g) d g \quad f \in \mathcal{H} G
$$

This operation of integration gives an equivalence of categories

$$
\left(\begin{array}{c}
\text { Smooth } \\
\text { representations of } G
\end{array}\right) \cong\left(\begin{array}{c}
\text { Non - degenerate } \\
\text { representations of } \mathcal{H} G
\end{array}\right)
$$

In particular this gives a bijection (of sets)

$$
\operatorname{Irr}(G) \longleftrightarrow \operatorname{Prim}(\mathcal{H} G)
$$

where $\operatorname{Prim}(\mathcal{H} G)$ is the set of primitive ideals in $\mathcal{H} G$.

What has been gained from this bijection?

On $\operatorname{Prim}(\mathcal{H} G)$ we have a topology : the Jacobson topology.

If $S$ is a subset of $\operatorname{Prim}(\mathcal{H} G)$ then the closure $\bar{S}$ (in the Jacobson topology) of $S$ is

$$
\bar{S}=\left\{J \in \operatorname{Prim}(\mathcal{H} G) \mid J \supset \bigcap_{I \in S} I\right\} .
$$

Example. Let $X$ be an affine variety over $\mathbb{C} . \mathcal{O}(X)$ denotes the coordinate algebra of $X$. Prim $(\mathcal{O}(X))$ is the set of $\mathbb{C}$-rational points of $X$. The Jacobson topology is the Zariski topology.

Point set topology. In a topological space $W$ two points $w_{1}, w_{2}$ are in the same connected component if and only if whenever $U_{1}, U_{2}$ are two open sets of $W$ with $w_{1} \in U_{1}, w_{2} \in U_{2}$, and $U_{1} \cup U_{2}=W$, then $U_{1} \cap U_{2} \neq \emptyset$.

As a set, $W$ is the disjoint union of its connected components. If each connected component is both open and closed, then as a topological space $W$ is the disjoint union of its connected components.

$\operatorname{Irr}(G)=\operatorname{Prim}(\mathcal{H} G)$ (with the Jacobson topology) is the disjoint union of its connected components. Each connected component is both open and closed. The 
connected components of $\operatorname{Irr}(G)=\operatorname{Prim}(\mathcal{H} G)$ are known as the Bernstein components.

$\pi_{o} \operatorname{Prim}(\mathcal{H} G)$ denotes the set of connected components of $\operatorname{Irr}(G)=\operatorname{Prim}(\mathcal{H} G)$.

$\pi_{o} \operatorname{Prim}(\mathcal{H} G)$ is a countable set and has no further structure.

$\pi_{o} \operatorname{Prim}(\mathcal{H} G)$ is known as the Bernstein spectrum of $G$, and will be denoted $\mathfrak{B}(G)$.

$$
\mathfrak{B}(G):=\pi_{o} \operatorname{Prim}(\mathcal{H} G)
$$

For $\mathfrak{s} \in \mathfrak{B}(G)$, the Bernstein component of $\operatorname{Irr}(G)=\operatorname{Prim}(\mathcal{H} G)$ will be denoted $\operatorname{Irr}(G)_{\mathfrak{s}}$.

\section{Cuspidal Support Map - Tempered Dual}

The main problem in the representation theory of reductive $p$-adic groups is:

Problem. Given a connected reductive $p$-adic group $G$, describe the smooth dual $\operatorname{Irr}(G)=\operatorname{Prim}(\mathcal{H} G)$.

A solution of this problem should include descriptions of

(1) The tempered dual

(2) The cuspidal support maps

(3) The LL map $\alpha_{G}: \operatorname{Irr}(G) \longrightarrow\{$ Langlands parameters for $G\} /{ }^{L} G^{0}$

For (1), recall that a choice of (left-invariant) Haar measure for $G$ determines a measure, the Plancherel measure, on the unitary dual of $G$. The tempered dual of $G$ is the support of the Plancherel measure [16]. Equivalently, the tempered dual consists of those smooth irreducible representations of $G$ whose Harish-Chandra character is tempered. Let $C_{r}^{*} G$ be the reduced $C^{*}$ algebra of $G[16] . \mathcal{H} G$ is a dense $*$-subalgebra of $C_{r}^{*} G$ which is not holomorphically closed.

$$
\mathcal{H} G \subset C_{r}^{*} G
$$

Then the tempered dual of $G$ consists of those irreducible representations of $\mathcal{H} G$ which can be extended to give an irreducible representation (in the sense of $C^{*}$ algebras) of $C_{r}^{*} G$. The tempered dual of $G$ will be denoted $\operatorname{Irr}(G)_{\text {temp. }}$.

$$
\operatorname{Irr}(G)_{\text {temp }} \subset \operatorname{Irr}(G)
$$

For (2) Bernstein $[12,13,31]$ assigns to each $\mathfrak{s} \in \mathfrak{B}(G)=\pi_{o} \operatorname{Prim}(\mathcal{H} G)$ a complex torus $T_{\mathfrak{s}}$ and a finite group $W_{\mathfrak{s}}$ which acts on $T_{\mathfrak{s}}$ as automorphisms of the affine variety $T_{\mathfrak{s}}$. Here "complex torus" means an algebraic group $T$, defined over the complex numbers $\mathbb{C}$, such that there exists an isomorphism of algebraic groups

$$
T \cong \mathbb{C}^{\times} \times \mathbb{C}^{\times} \times \cdots \times \mathbb{C}^{\times} .
$$

In general, $W_{\mathfrak{s}}$ acts on $T_{\mathfrak{s}}$ not as automorphisms of the algebraic group $T_{\mathfrak{s}}$ but only as automorphisms of the affine variety $T_{\mathfrak{s}}$. Bernstein then forms the quotient variety $T_{\mathfrak{s}} / W_{\mathfrak{s}}$ and proves that there is a surjective map (of sets) $\pi_{\mathfrak{s}}$ mapping the 
Bernstein component $\operatorname{Irr}(G)_{\mathfrak{s}}$ onto $T_{\mathfrak{s}} / W_{\mathfrak{s}}$. This map $\pi_{\mathfrak{s}}$ is known as the infinitesimal central character or the cuspidal support map.

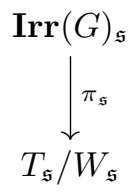

The cuspidal support map encodes essential information about the representation theory of $G$. Thus any description of $\operatorname{Irr}(G)_{\mathfrak{s}}$ which did not include a calculation of the cuspidal support map would be very incomplete.

Remark. $T_{\mathfrak{s}}$ is an algebraic group, defined over $\mathbb{C}$, which as an algebraic group is non-canonically isomorphic to $\mathbb{C}^{\times} \times \mathbb{C}^{\times} \times \cdots \times \mathbb{C}^{\times}$.

$$
T_{\mathfrak{s}} \cong \mathbb{C}^{\times} \times \mathbb{C}^{\times} \times \cdots \times \mathbb{C}^{\times}
$$

Denote the maximal compact subgroup of $T_{\mathfrak{s}}$ by $T_{\mathfrak{s}}^{\mathrm{cpt}}$. Although the action of $W_{\mathfrak{s}}$ on $T_{\mathfrak{s}}$ in general is not as automorphisms of the algebraic group $T_{\mathfrak{s}}$ (but only as automorphisms of the affine variety $T_{\mathfrak{s}}$ ), this action always preserves $T_{\mathfrak{s}}^{\mathrm{cpt}}$.

$$
W_{\mathfrak{s}} \times T_{\mathfrak{s}}^{\mathrm{cpt}} \longrightarrow T_{\mathfrak{s}}^{\mathrm{cpt}}
$$

$T_{\mathfrak{s}}, W_{\mathfrak{s}}$ and the action

$$
W_{\mathfrak{s}} \times T_{\mathfrak{s}} \longrightarrow T_{\mathfrak{s}}
$$

of $W_{\mathfrak{s}}$ on $T_{\mathfrak{s}}$ are usually quite easily calculated. $\operatorname{Irr}(G)_{\mathfrak{s}}$ and the cuspidal support map $\pi_{\mathfrak{s}}$ can be (and very often are) extremely difficult to describe and calculate.

The ABPS conjecture states that $\operatorname{Irr}(G)_{\mathfrak{s}}$ has a very simple geometric structure given by the extended quotient.

\section{Extended Quotient}

Let $\Gamma$ be a finite group acting on a complex affine variety $X$ as automorphisms of the affine variety

$$
\Gamma \times X \rightarrow X .
$$

The quotient variety $X / \Gamma$ is obtained by collapsing each orbit to a point. $X / \Gamma$ is an affine variety.

For $x \in X, \Gamma_{x}$ denotes the stabilizer group of $x$,

$$
\Gamma_{x}=\{\gamma \in \Gamma: \gamma x=x\} .
$$

$c\left(\Gamma_{x}\right)$ denotes the set of conjugacy classes of $\Gamma_{x}$. The extended quotient is obtained by replacing the orbit of $x$ by $c\left(\Gamma_{x}\right)$. This is done as follows:

Set $\widetilde{X}=\{(\gamma, x) \in \Gamma \times X: \gamma x=x\} . \tilde{X}$ is an affine variety and is a subvariety of $\Gamma \times X$. The group $\Gamma$ acts on $\tilde{X}$ :

$$
\begin{gathered}
\Gamma \times \widetilde{X} \rightarrow \widetilde{X} \\
\alpha(\gamma, x)=\left(\alpha \gamma \alpha^{-1}, \alpha x\right), \quad \alpha \in \Gamma, \quad(\gamma, x) \in \widetilde{X} .
\end{gathered}
$$


The extended quotient, denoted $X / / \Gamma$, is $\widetilde{X} / \Gamma$. Thus the extended quotient $X / / \Gamma$ is the usual quotient for the action of $\Gamma$ on $\widetilde{X}$. We remark that this differs from the quotient in geometric invariant theory.

The projection $\widetilde{X} \rightarrow X,(\gamma, x) \mapsto x$ is $\Gamma$-equivariant and so passes to quotient spaces to give a morphism of affine varieties

$$
\rho: X / / \Gamma \rightarrow X / \Gamma
$$

This map will be referred to as the projection of the extended quotient onto the ordinary quotient.

The inclusion

$$
\begin{aligned}
X \hookrightarrow \tilde{X} & \\
x & \mapsto(e, x) \quad e=\text { identity element of } \Gamma
\end{aligned}
$$

is $\Gamma$-equivariant and so passes to quotient spaces to give an inclusion of affine varieties $X / \Gamma \hookrightarrow X / / \Gamma$. This will be referred to as the inclusion of the ordinary quotient in the extended quotient.

Notation. $X / / \Gamma$ with $X / \Gamma$ removed will be denoted $X / / \Gamma-X / \Gamma$.

\section{Approximate Statement of the ABps conjecture}

Conjecture. Let $G$ be a connected reductive $p$-adic group. Assume that $G$ is quasisplit or that $G$ is an inner form of $\operatorname{GL}(n, F)$. Let $\operatorname{Irr}(G)_{\mathfrak{s}}$ be a Bernstein component of $\operatorname{Irr}(G)$. Let $T_{\mathfrak{s}}$ and $W_{\mathfrak{s}}$ be the complex torus and finite group [12, 13, 31] assigned by Bernstein to $\mathfrak{s} \in \mathfrak{B}(G)$. Denote by $\pi_{\mathfrak{s}}: \operatorname{Irr}(G)_{\mathfrak{s}} \rightarrow T_{\mathfrak{s}} / W_{\mathfrak{s}}$ and $\rho_{\mathfrak{s}}: T_{\mathfrak{s}} / / W_{\mathfrak{s}} \rightarrow$ $T_{\mathfrak{s}} / W_{\mathfrak{s}}$ the cuspidal support map and the projection of the extended quotient onto the ordinary quotient. Then :

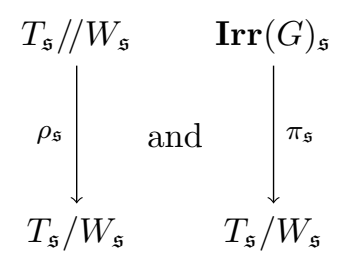

are almost the same.

As indicated above, $\operatorname{Irr}(G)_{\mathfrak{s}}$ and the cuspidal support map $\pi_{\mathfrak{s}}: \operatorname{Irr}(G)_{\mathfrak{s}} \longrightarrow T_{\mathfrak{s}} / W_{\mathfrak{s}}$ are often very difficult to describe and calculate. The extended quotient $T_{\mathfrak{s}} / / W_{\mathfrak{s}}$ and its projection $\rho_{\mathfrak{s}}: T_{\mathfrak{s}} / / W_{\mathfrak{s}} \longrightarrow T_{\mathfrak{s}} / W_{\mathfrak{s}}$ onto the ordinary quotient are usually quite easily calculated.

What is the mathematical meaning of "are almost the same"? The precise statement of the ABPS conjecture will be given in Section 15 and uses the extended quotient of the second kind.

\section{Extended QUOTIENT OF THE SECOND KIND}

Let $\Gamma$ be a finite group acting as automorphisms of a complex affine variety $X$.

$$
\Gamma \times X \rightarrow X
$$


For $x \in X, \Gamma_{x}$ denotes the stabilizer group of $x$ :

$$
\Gamma_{x}=\{\gamma \in \Gamma: \gamma x=x\} .
$$

Let $\operatorname{Irr}\left(\Gamma_{x}\right)$ be the set of (equivalence classes of) irreducible representations of $\Gamma_{x}$. These representations are on finite dimensional vector spaces over the complex numbers $\mathbb{C}$.

The extended quotient of the second kind, denoted $(X / / \Gamma)_{2}$, is constructed by replacing the orbit of $x$ (for the given action of $\Gamma$ on $X$ ) by $\operatorname{Irr}\left(\Gamma_{x}\right)$. This is done as follows :

Set $\widetilde{X}_{2}=\left\{(x, \tau) \mid x \in X\right.$ and $\left.\tau \in \operatorname{Irr}\left(\Gamma_{x}\right)\right\}$. Endowed with the topology that sees only the first coordinate, this is an algebraic variety in the sense of [? ], although it is usually not separated. Then $\Gamma$ acts on $\widetilde{X}_{2}$ by

$$
\begin{aligned}
& \Gamma \times \widetilde{X}_{2} \rightarrow \widetilde{X}_{2}, \\
& \gamma(x, \tau)=\left(\gamma x, \gamma_{*} \tau\right),
\end{aligned}
$$

where $\gamma_{*}: \operatorname{Irr}\left(\Gamma_{x}\right) \rightarrow \operatorname{Irr}\left(\Gamma_{\gamma x}\right) .(X / / \Gamma)_{2}$ is defined by:

$$
(X / / \Gamma)_{2}:=\widetilde{X}_{2} / \Gamma
$$

i.e. $(X / / \Gamma)_{2}$ is the usual quotient for the action of $\Gamma$ on $\widetilde{X}_{2}$.

The projection $\widetilde{X}_{2} \rightarrow X \quad(x, \tau) \mapsto x \quad$ is $\Gamma$-equivariant and so passes to quotient spaces to give the projection of $(X / / \Gamma)_{2}$ onto $X / \Gamma$.

$$
\rho_{2}:(X / / \Gamma)_{2} \longrightarrow X / \Gamma
$$

Denote by $\operatorname{triv}_{x}$ the trivial one-dimensional representation of $\Gamma_{x}$. The inclusion

$$
\begin{aligned}
X & \hookrightarrow \widetilde{X}_{2} \\
x & \mapsto\left(x, \operatorname{triv}_{x}\right)
\end{aligned}
$$

is $\Gamma$-equivariant and so passes to quotient spaces to give an inclusion

$$
X / \Gamma \hookrightarrow(X / / \Gamma)_{2}
$$

This will be referred to as the inclusion of the ordinary quotient in the extended quotient of the second kind.

Let $\mathcal{O}(X)$ be the coordinate algebra of the complex affine variety $X$ and let $\mathcal{O}(X) \rtimes \Gamma$ be the crossed-product algebra for the action of $\Gamma$ on $\mathcal{O}(X)$. There are canonical bijections

$$
\operatorname{Irr}(\mathcal{O}(X) \rtimes \Gamma) \longleftrightarrow \operatorname{Prim}(\mathcal{O}(X) \rtimes \Gamma) \longleftrightarrow(X / / \Gamma)_{2},
$$

where $\operatorname{Prim}(\mathcal{O}(X) \rtimes \Gamma)$ is the set of primitive ideals in $\mathcal{O}(X) \rtimes \Gamma$ and $\operatorname{Irr}(\mathcal{O}(X) \rtimes \Gamma)$ is the set of (equivalence classes of) irreducible representations of $\mathcal{O}(X) \rtimes \Gamma$. The irreducible representation of $\mathcal{O}(X) \rtimes \Gamma$ associated to $(x, \tau) \in(X / / \Gamma)_{2}$ is

$$
\operatorname{Ind}_{\mathcal{O}(X) \rtimes \Gamma_{x}}^{\mathcal{O}(X) \rtimes \Gamma}\left(\mathbb{C}_{x} \otimes \tau\right) .
$$

Here $\mathbb{C}_{x}: \mathcal{O}(X) \rightarrow \mathbb{C}$ is the irreducible representation of $\mathcal{O}(X)$ given by evaluation at $x \in X$. $\operatorname{Ind}_{\mathcal{O}(X) \rtimes \Gamma \Gamma_{x}}^{\mathcal{O}(X) \rtimes \Gamma}$ is induction from $\mathcal{O}(X) \rtimes \Gamma_{x}$ to $\mathcal{O}(X) \rtimes \Gamma$.

$\operatorname{Prim}(\mathcal{O}(X) \rtimes \Gamma)$ is endowed with the Jacobson topology, which makes it a (not 
necessarily separated) algebraic variety. This structure can be transferred via the canonical bijection $\operatorname{Prim}(\mathcal{O}(X) \rtimes \Gamma) \longleftrightarrow(X / / \Gamma)_{2}$ to $(X / / \Gamma)_{2}$. Hence $(X / / \Gamma)_{2}$ is a complex algebraic variety. In many examples $(X / / \Gamma)_{2}$ is not separated, and is not an affine variety.

Example 1. Let $X=\mathbb{C}^{\times}$and $\Gamma=\mathbb{Z} / 2 \mathbb{Z}$ where the generator of $\mathbb{Z} / 2 \mathbb{Z}$ acts by $\zeta \mapsto \zeta^{-1}$. The stabilizers are trivial except at 1 and -1 where the stablizer is $\mathbb{Z} / 2 \mathbb{Z}$. Each fiber of the map $\rho_{2}:(X / / \Gamma)_{2} \rightarrow X / \Gamma$ consists of just one point except for two fibers which consist of 2 points. Hence $(X / / \Gamma)_{2}$ is $X / \Gamma$ with 2 double points. Each double point cannot be separated within the Jacobson topology, i.e. $(X / / \Gamma)_{2}$ is not a $T_{1}$-space. Hence in this example $(X / / \Gamma)_{2}$ is a nonseparated algebraic variety.

From the non-commutative geometry point of view, $\mathcal{O}(X) \rtimes \Gamma$ is the coordinate algebra of the non-commutative affine algebraic variety $(X / / \Gamma)_{2}$.

\section{Comparison of the two extended quotients}

With $X, \Gamma$ as above, there is a non-canonical bijection $\epsilon: X / / \Gamma \rightarrow(X / / \Gamma)_{2}$ with commutativity in the diagrams
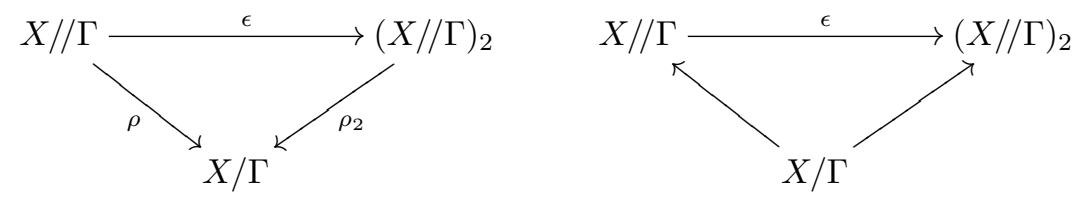

The map $\epsilon$ is a morphism of nonseparated algebraic varieties. In most examples $\epsilon^{-1}$ is not continuous. Hence, in most examples (e.g. Example 1), the map $\epsilon$ is not an isomorphism of algebraic varieties and is not a homeomorphism.

To construct the non-canonical bijection $\epsilon$, some choices must be made. Let $\psi$ be a family of bijections (one bijection $\psi_{x}$ for each $x \in X$ )

$$
\psi_{x}: c\left(\Gamma_{x}\right) \rightarrow \operatorname{Irr}\left(\Gamma_{x}\right)
$$

such that for all $x \in X$ :

(1) $\psi_{x}(e)=\operatorname{triv}_{x} \quad e=$ identity element of $\Gamma_{x}$

(2) $\psi_{\gamma x}\left(\left[\gamma g \gamma^{-1}\right]\right)=\phi_{x}([g]) \circ \operatorname{Ad}_{\gamma}^{-1}$ for all $g \in \Gamma_{x}, \gamma \in \Gamma$

(3) $\psi_{x}=\psi_{y}$ if $\Gamma_{x}=\Gamma_{y}$ and $x, y$ belong to the same connected component of the variety $X^{\Gamma_{x}}:=\left\{z \in X \mid \gamma z=z \forall \gamma \in \Gamma_{x}\right\}$

Such a family of bijections will be referred to as a $c$-Irr system. $\psi$ induces a map $\widetilde{X} \rightarrow \widetilde{X}_{2}$ which preserves the $X$-coordinates. By property (2) this map is $\Gamma$-equivariant, so it descends to give a bijection

$$
\epsilon=\epsilon_{\psi}: X / / \Gamma \rightarrow(X / / \Gamma)_{2} .
$$

such that the diagrams (1) commute. Property (3) is not really needed, but serves to exclude some rather awkward and unpleasant choices of $\psi$.

As remarked above, the crossed-product algebra $\mathcal{O}(X) \rtimes \Gamma$ can be viewed as the coordinate algebra of the non-commutative affine algebraic variety $(X / / \Gamma)_{2}$.

There are some intriguing similarities and differences between the two finite-type $\mathcal{O}(X / \Gamma)$-algebras $\mathcal{O}(X / / \Gamma)$ and $\mathcal{O}(X) \rtimes \Gamma$. In many examples (e.g. if $X$ is connected and the action of $\Gamma$ on $X$ is neither trivial nor free) these two algebras are not 
Morita equivalent. However, these two algebras always have the same periodic cyclic homology:

$$
H P_{*}(\mathcal{O}(X) \rtimes \Gamma) \cong H P_{*}(\mathcal{O}(X / / \Gamma)) \cong H^{*}(X / / \Gamma ; \mathbb{C}) .
$$

$H^{*}(X / / \Gamma ; \mathbb{C})$ is the cohomology (in the usual sense of algebraic topology), with coefficients $\mathbb{C}$, of the underlying locally compact Hausdorff space of the complex affine variety $X / / \Gamma$.

In the example relevant to the representation theory of reductive $p$-adic groups (i.e. $\Gamma=W_{\mathfrak{s}}, X=T_{\mathfrak{s}}$ ) these two finite-type $\mathcal{O}(X / \Gamma)$-algebras are very often conjecturally always - equivalent via a weakening of Morita equivalence referred to as "geometric equivalence". See the appendix for the definition of "geometric equivalence".

The finite group $W_{\mathfrak{s}}$ is often an extended finite Coxeter group i.e. $W_{\mathfrak{s}}$ is often a semi-direct product for the action of a finite abelian group $A$ on a finite Weyl group $W$ :

$$
W_{\mathfrak{s}}=W \rtimes A .
$$

Due to this, in many examples there is a clear preferred choice of $c$-Irr system for the action of $W_{\mathfrak{s}}$ on $T_{\mathfrak{s}}$.

Example. For the groups $\mathrm{GL}(m, D)$ every Bernstein component has the two extended quotients $T_{\mathfrak{s}} / / W_{\mathfrak{s}}$ and $\left(T_{\mathfrak{s}} / / W_{\mathfrak{s}}\right)_{2}$ canonically in bijection. The reason for this is that all the stabilizer groups for the action of $W_{\mathfrak{s}}$ on $T_{\mathfrak{s}}$ are finite Cartesian products of symmetric groups:

$$
W_{\mathfrak{s}, t} \cong \prod_{i} S_{i}
$$

The classical theory of Young tableaux (or the Springer correspondence) then applies to give a canonical bijection between $\operatorname{Irr}\left(\prod_{i} S_{i}\right)$ and the set of conjugacy classes in $\prod_{i} S_{i}$ - i.e. a canonical c-Irr system for the action of $W_{\mathfrak{s}}$ on $T_{\mathfrak{s}}$.

Remark. If $S$ is any set and $\Gamma$ is any group acting on $S$, then (in an evident way) the two extended quotients $S / / \Gamma$ and $(S / / \Gamma)_{2}$ can be formed.

\section{Statement of the ABPS conjecture}

Let $G$ be a connected reductive p-adic group. Assume that $G$ is quasi-split or that $G$ is an inner form of $\mathrm{GL}(n, F)$. Let $\mathfrak{s}$ be a point in the Bernstein spectrum of $G$.

$$
\mathfrak{s} \in \mathfrak{B}(G)=\pi_{o} \operatorname{Prim}(\mathcal{H} G)
$$

Recall that $T_{\mathfrak{s}} / / W_{\mathfrak{s}}$ and $\left(T_{\mathfrak{s}} / / W_{\mathfrak{s}}\right)_{2}$ are the extended quotients of the first and second kind defined in $\S 11$ and $\S 13$.

The statement of the ABPS conjecture given in this section explains the meaning of the phrase "almost the same" in $\S 12$.

The ABPS conjecture [3]-[7] consists of the following five statements.

(1) The cuspidal support map

$$
\pi_{\mathfrak{s}}: \operatorname{Irr}(G)_{\mathfrak{s}} \rightarrow T_{\mathfrak{s}} / W_{\mathfrak{s}}
$$

is one-to-one if and only if the action of $W_{\mathfrak{s}}$ on $T_{\mathfrak{s}}$ is free. 
(2) There is a canonically defined commutative triangle

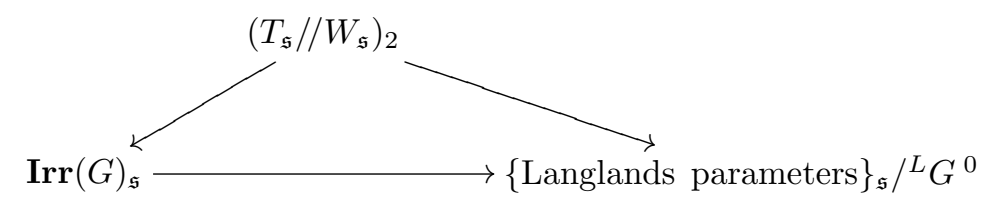

in which the left slanted arrow is bijective and the horizontal arrow is the map of the local Langlands correspondence.

$\{\text { Langlands parameters }\}_{\mathfrak{s}}$ is those Langlands parameters whose L-packets have non-empty intersection with $\operatorname{Irr}(G)_{\mathfrak{s}}$.

The maps in this commutative triangle are canonical.

(3) The canonical bijection

$$
\left(T_{\mathfrak{s}} / / W_{\mathfrak{s}}\right)_{2} \longrightarrow \operatorname{Irr}(G)_{\mathfrak{s}}
$$

comes from a canonical geometric equivalence of the two unital finite-type $\mathcal{O}\left(T_{\mathfrak{s}} / W_{\mathfrak{s}}\right)$-algebras $\mathcal{O}\left(T_{\mathfrak{s}}\right) \rtimes W_{\mathfrak{s}}$ and $\mathcal{H}_{\mathfrak{s}}$. See the appendix for details on "geometric equivalence".

(4) The canonical bijection

$$
\left(T_{\mathfrak{s}} / / W_{\mathfrak{s}}\right)_{2} \longrightarrow \operatorname{Irr}(G)_{\mathfrak{s}}
$$

maps $\left(T_{\mathfrak{s}}^{\mathrm{cpt}} / / W_{\mathfrak{s}}\right)_{2}$ onto $\operatorname{Irr}(G)_{\mathfrak{s}} \cap \operatorname{Irr}(G)_{\text {temp }}$.

(5) A $c$-Irr system can be chosen for the action of $W_{\mathfrak{s}}$ on $T_{\mathfrak{s}}$ such that the resulting (non-canonical) bijection

$$
\epsilon: T_{\mathfrak{s}} / / W_{\mathfrak{s}} \longrightarrow\left(T_{\mathfrak{s}} / / W_{\mathfrak{s}}\right)_{2}
$$

when composed with the canonical bijection $\left(T_{\mathfrak{s}} / / W_{\mathfrak{s}}\right)_{2} \rightarrow \operatorname{Irr}(G)_{\mathfrak{s}}$ gives a bijection

$$
\mu_{\mathfrak{s}}: T_{\mathfrak{s}} / / W_{\mathfrak{s}} \longrightarrow \operatorname{Irr}(G)_{\mathfrak{s}}
$$

which has the following six properties:

Notation for Property 1:

Within the smooth dual $\operatorname{Irr}(G)$, there is the tempered dual

$\operatorname{Irr}(G)_{\text {temp }}=\{$ smooth tempered irreducible representations of $G\} / \sim$

$T_{\mathfrak{s}}^{\mathrm{cpt}}=$ maximal compact subgroup of $T_{\mathfrak{s}}$.

$T_{\mathfrak{s}}^{\mathrm{cpt}}$ is a compact real torus. The action of $W_{\mathfrak{s}}$ on $T_{\mathfrak{s}}$ preserves $T_{\mathfrak{s}}^{\mathrm{cpt}}$, so the extended quotient $T_{\mathfrak{s}}^{\mathrm{cpt}} / / W_{\mathfrak{s}}$ can be formed.

Property 1 of the bijection $\mu_{\mathfrak{s}}$ :

The bijection $\mu_{\mathfrak{s}}: T_{\mathfrak{s}} / / W_{\mathfrak{s}} \longrightarrow \operatorname{Irr}(G)_{\mathfrak{s}}$ maps $T_{\mathfrak{s}}^{\mathrm{cpt}} / / W_{\mathfrak{s}}$ onto $\operatorname{Irr}(G)_{\mathfrak{s}} \cap \operatorname{Irr}(G)_{\text {temp }}$, and hence restricts to a bijection

$$
\mu_{\mathfrak{s}}: T_{\mathfrak{s}}^{\mathrm{cpt}} / / W_{\mathfrak{s}} \longleftrightarrow \operatorname{Irr}(G)_{\mathfrak{s}} \cap \operatorname{Irr}(G)_{\mathrm{temp}}
$$

Property 2 of the bijection $\mu_{\mathfrak{s}}$ : 
For many $\mathfrak{s} \in \mathfrak{B}(G)$ the diagram

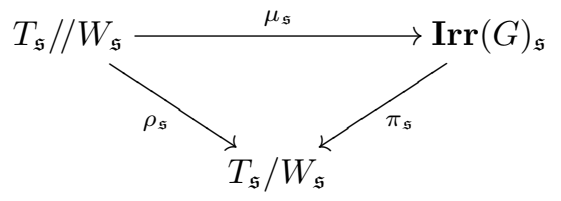

does not commute.

Property 3 of the bijection $\mu_{\mathfrak{s}}$ :

In the possibly non-commutative diagram

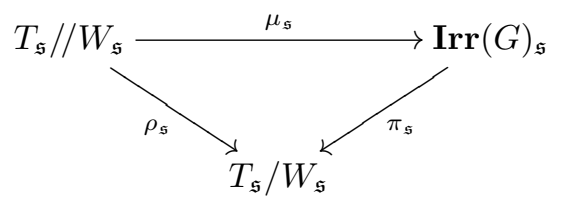

the bijection $\mu_{\mathfrak{s}}: T_{\mathfrak{s}} / / W_{\mathfrak{s}} \longrightarrow \operatorname{Irr}(G)_{\mathfrak{s}}$ is continuous, where the affine variety $T_{\mathfrak{s}} / / W_{\mathfrak{s}}$ has the Zariski topology and $\operatorname{Irr}(G)_{\mathfrak{s}} \subset \operatorname{Prim}(\mathcal{H} G)$ has the Jacobson topology and the composition

$$
\pi_{\mathfrak{s}} \circ \mu_{\mathfrak{s}}: T_{\mathfrak{s}} / / W_{\mathfrak{s}} \longrightarrow T_{\mathfrak{s}} / W_{\mathfrak{s}}
$$

is a morphism of complex affine algebraic varieties.

Property 4 of the bijection $\mu_{\mathfrak{s}}$ :

There is an algebraic family

$$
\theta_{z}: T_{\mathfrak{s}} / / W_{\mathfrak{s}} \longrightarrow T_{\mathfrak{s}} / W_{\mathfrak{s}}
$$

of finite morphisms of algebraic varieties, with $z \in \mathbb{C}^{\times}$, such that

$$
\theta_{1}=\rho_{\mathfrak{s}}, \quad \theta_{\sqrt{q}}=\pi_{\mathfrak{s}} \circ \mu_{\mathfrak{s}}
$$

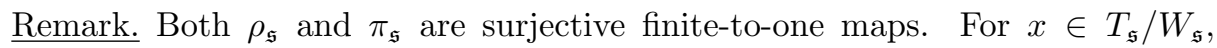
denote by $\#\left(x, \rho_{\mathfrak{s}}\right), \#\left(x, \pi_{\mathfrak{s}}\right)$ the number of points in the pre-image of $x$ using $\rho_{\mathfrak{s}}$, $\pi_{\mathfrak{s}}$. The numbers $\#\left(x, \pi_{\mathfrak{s}}\right)$ are of interest in representation theory. Within $T_{\mathfrak{s}} / W_{\mathfrak{s}}$ the algebraic sub-varieties $R\left(\rho_{\mathfrak{s}}\right), R\left(\pi_{\mathfrak{s}}\right)$ are defined by

$$
\begin{aligned}
& R\left(\rho_{\mathfrak{s}}\right):=\left\{x \in T_{\mathfrak{s}} / W_{\mathfrak{s}} \mid \#\left(x, \rho_{\mathfrak{s}}\right)>1\right\} \\
& R\left(\pi_{\mathfrak{s}}\right):=\left\{x \in T_{\mathfrak{s}} / W_{\mathfrak{s}} \mid \#\left(x, \pi_{\mathfrak{s}}\right)>1\right\}
\end{aligned}
$$

Setting

$$
Y_{z}=\theta_{z}\left(T_{\mathfrak{s}} / / W_{\mathfrak{s}}-T_{\mathfrak{s}} / W_{\mathfrak{s}}\right)
$$

a flat family of sub-schemes of $T_{\mathfrak{s}} / W_{\mathfrak{s}}$ is obtained with

$$
Y_{1}=R\left(\rho_{\mathfrak{s}}\right), \quad Y_{\sqrt{q}}=R\left(\pi_{\mathfrak{s}}\right) .
$$

Property 5 of the bijection $\mu_{\mathfrak{s}}$ (Correcting cocharacters):

For each connected component $\mathbf{c}$ of the affine variety $T_{\mathfrak{s}} / / W_{\mathfrak{s}}$ there is a cocharacter (i.e. a homomorphism of algebraic groups)

$$
h_{c}: \mathbb{C}^{\times} \longrightarrow T_{\mathfrak{s}}
$$

such that

$$
\theta_{z}[w, t]=b\left(h_{c}(z) \cdot t\right)
$$


for all $[w, t] \in \mathbf{c}$.

Let $b: T_{\mathfrak{s}} \longrightarrow T_{\mathfrak{s}} / W_{\mathfrak{s}}$ be the quotient map. Here, as above, points of $\widetilde{T}_{\mathfrak{s}}$ are pairs $(w, t)$ with $w \in W_{\mathfrak{s}}, t \in T_{\mathfrak{s}}$ and $w t=t$. $[w, t]$ is the point in $T_{\mathfrak{s}} / / W_{\mathfrak{s}}$ obtained by applying the quotient map $\widetilde{T}_{\mathfrak{s}} \rightarrow T_{\mathfrak{s}} / / W_{\mathfrak{s}}$ to $(w, t)$.

Remark. The equality

$$
\theta_{z}[w, t]=b\left(h_{c}(z) \cdot t\right)
$$

is to be interpreted thus:

Let $Z_{1}, Z_{2}, \ldots, Z_{r}$ be the connected components of the affine variety $T_{\mathfrak{s}} / / W_{\mathfrak{s}}$ and let $h_{1}, h_{2}, \ldots, h_{r}$ be the cocharacters as in the statement of Property 5 . Let

$$
\nu_{\mathfrak{s}}: \widetilde{T_{\mathfrak{s}}} \longrightarrow T_{\mathfrak{s}} / / W_{\mathfrak{s}}
$$

be the quotient map.

Then connected components $X_{1}, X_{2}, \ldots, X_{r}$ of the affine variety $\widetilde{T_{\mathfrak{s}}}$ can be chosen with

- $\nu_{\mathfrak{s}}\left(X_{j}\right)=Z_{j}$ for $j=1,2, \ldots, r$

- For each $z \in \mathbb{C}^{\times}$the map $m_{z}: X_{j} \rightarrow T_{\mathfrak{s}} / W_{\mathfrak{s}}$, which is the composition

$$
\begin{gathered}
X_{j} \longrightarrow T_{\mathfrak{s}} \longrightarrow T_{\mathfrak{s}} / W_{\mathfrak{s}} \\
(w, t) \longmapsto h_{j}(z) t \longmapsto b\left(h_{j}(z) t\right),
\end{gathered}
$$

makes the diagram

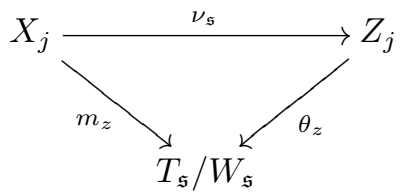

commutative. Note that $h_{j}(z) t$ is the product of $h_{j}(z)$ and $t$ in the algebraic group $T_{\mathfrak{s}}$.

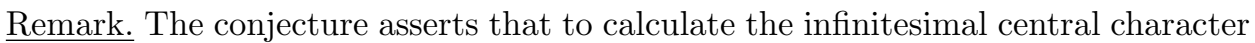

$$
\pi_{\mathfrak{s}}: \widehat{G}_{\mathfrak{s}} \longrightarrow T_{\mathfrak{s}} / W_{\mathfrak{s}}
$$

two steps suffice:

Step 1: Calculate the projection of the extended quotient onto the ordinary quotient

$$
\rho_{\mathfrak{s}}: T_{\mathfrak{s}} / / W_{\mathfrak{s}} \longrightarrow T_{\mathfrak{s}} / W_{\mathfrak{s}}
$$

Step 2: Determine the correcting cocharacters.

The cocharacter assigned to $T_{\mathfrak{s}} / W_{\mathfrak{s}} \hookrightarrow T_{\mathfrak{s}} / / W_{\mathfrak{s}}$ is always the trivial cocharacter mapping $\mathbb{C}^{\times}$to the unit element of $T_{\mathfrak{s}}$. So all the non-trivial correcting is taking place on $T_{\mathfrak{s}} / / W_{\mathfrak{s}}-T_{\mathfrak{s}} / W_{\mathfrak{s}}$.

Notation for Property 6.

If $S$ and $V$ are sets, a labelling of $S$ by $V$ is a map of sets $\lambda: S \rightarrow V$.

Property 6 of the bijection $\mu_{\mathfrak{s}}$ ( $L$-packets):

As in Property 5 , let $\left\{Z_{1}, \ldots, Z_{r}\right\}$ be the irreducible components of the affine variety $T_{\mathfrak{s}} / / W_{\mathfrak{s}}$, and let $\left\{h_{1}, h_{2}, \ldots, h_{r}\right\}$ be the correcting cocharacters. 
Then a finite set $V$ and a labelling $\lambda:\left\{Z_{1}, Z_{2}, \ldots, Z_{r}\right\} \rightarrow V$ exist such that for every two points $[w, t]$ and $\left[w^{\prime}, t^{\prime}\right]$ of $T_{\mathfrak{s}} / / W_{\mathfrak{s}}$ :

$$
\mu_{\mathfrak{s}}[w, t] \text { and } \mu_{\mathfrak{s}}\left[w^{\prime}, t^{\prime}\right] \text { are in the same } L \text {-packet }
$$

if and only

(i) $\theta_{z}[w, t]=\theta_{z}\left[w^{\prime}, t^{\prime}\right]$ for all $z \in \mathbb{C}^{\times}$;

(ii) $\lambda[w, t]=\lambda\left[w^{\prime}, t^{\prime}\right]$, where $\lambda$ has been lifted to a labelling of $T_{\mathfrak{s}} / / W_{\mathfrak{s}}$ in the evident way.

Remark. An $L$-packet can have non-empty intersection with more than one Bernstein component. The conjecture does not address this issue. The conjecture only describes the intersections of $L$-packets with any one given Bernstein component.

In brief, the conjecture asserts that - once a Bernstein component has been fixed - intersections of $L$-packets with that Bernstein component consisting of more than one point are "caused" by repetitions among the correcting cocharacters. If, for any one given Bernstein component, the correcting cocharacters $h_{1}, h_{2}, \ldots, h_{r}$ are all distinct, then (according to the conjecture) the intersections of $L$-packets with that Bernstein component are singletons.

A Langlands parameter

$$
\mathcal{W}_{F} \times \mathrm{SL}(2, \mathbb{C}) \longrightarrow{ }^{L} G
$$

determines a homomorphism of complex algebraic groups

$$
\operatorname{SL}(2, \mathbb{C}) \longrightarrow{ }^{L} G^{0}
$$

Let $T\left({ }^{L} G^{0}\right)$ be the maximal torus of ${ }^{L} G^{0}$. By restricting the homomorphism of complex algebraic groups $\mathrm{SL}(2, \mathbb{C}) \rightarrow{ }^{L} G^{0}$ to the maximal torus of $\mathrm{SL}(2, \mathbb{C})$ a cocharacter

$$
\mathbb{C}^{\times} \longrightarrow T\left({ }^{L} G^{0}\right)
$$

is obtained. In examples, these are the correcting cocharacters.

\section{Two Theorems}

As in $\S 8$, let $D$ be a division algebra of dimension $d^{2}$ over its center $F$. With $m$ a positive integer, $\operatorname{GL}(m, D)$ denotes the connected reductive $p$-adic group consisting of all $m \times m$ invertible matrices with entries in $D$. Except for $\operatorname{GL}(n, F)$, these groups are non-split. $\mathrm{GL}(m, D)$ is an inner form of $\mathrm{GL}(m d, F)$. Hence all the groups $\mathrm{GL}(m, D)$ are inner forms of connected split reductive $p$-adic groups.

Theorem 1. The ABPS conjecture is valid for $G=\mathrm{GL}(m, D)$. In this case, for each Bernstein component $\operatorname{Irr}(G)_{\mathfrak{s}} \subset \operatorname{Irr}(G)$, all three maps in the commutative triangle

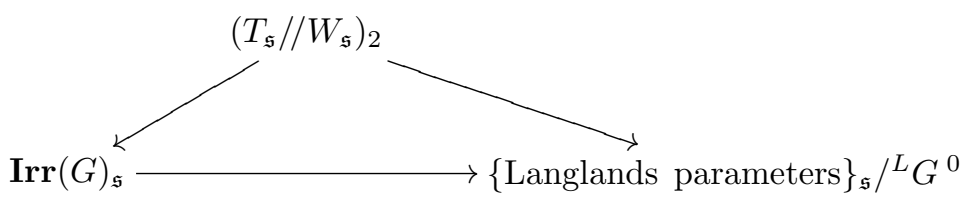

are bijective. 
Assume now that $G$ is connected and split.

As in section 7 above, let $\mathbb{T}$ be a maximal $p$-adic torus in $G$ and let $B$ be a Borel subgroup of $G$ containing $\mathbb{T}$. An irreducible smooth representation $\phi$ of $G$

$$
\phi: G \rightarrow \operatorname{Aut}_{\mathbb{C}}(V)
$$

is in the principal series if and only if there exists a smooth irreducible representation $\chi$ of $\mathbb{T}$, i.e. a smooth character

$$
\chi: \mathbb{T} \longrightarrow \mathbb{C}^{\times}
$$

with $\phi$ a sub-quotient of $\operatorname{Ind}_{B}^{G}(\chi)$. Here $\operatorname{Ind}_{B}^{G}$ is the (normalized) smooth parabolic induction from $\mathbb{T}$ to $G$ via $B$.

The notion principal series does not depend on the choice of $\mathbb{T}$ or $B$. Any Bernstein component $\operatorname{Irr}(G)_{\mathfrak{s}}$ in $\operatorname{Irr}(G)$ either has empty intersection with the principal series or is completely contained within the principal series.

The Langlands parameters

$$
\varphi: \mathcal{W}_{F} \times \operatorname{SL}(2, \mathbb{C}) \longrightarrow^{L} G^{0}
$$

for the principal series have a very simple form. They are those Langlands parameters $\varphi$ such that when restricted to $\mathcal{W}_{F}, \varphi$ is trivial on $\mathcal{W}_{F}^{\text {der }}$ - i.e. the restriction of $\varphi$ to $\mathcal{W}_{F}$ factors through $\mathcal{W}_{F}^{\mathrm{ab}}$.

$$
\mathcal{W}_{F}^{\mathrm{ab}}:=\mathcal{W}_{F} / \mathcal{W}_{F}^{\text {der }}
$$

Due to the isomorphism of local class field theory

$$
\mathcal{W}_{F}^{\mathrm{ab}} \cong F^{\times}
$$

such a Langlands parameter $\varphi$ can be viewed as a continuous group homomorphism

$$
\varphi: F^{\times} \times \mathrm{SL}(2, \mathbb{C}) \longrightarrow{ }^{L} G^{\circ} \text {. }
$$

An enhancement $[25,22,1,34]$ of such a $\varphi$ is a pair $(\varphi, \sigma)$ where $\sigma$ is an irreducible representation of the finite group $\pi_{0}\left(Z_{L} G_{0}(\right.$ Image $\left.\varphi)\right)$ which occurs in $H^{*}(\mathcal{B} \mathcal{V}(\varphi) ; \mathbb{C})$. The notation is:

Notation.

- Image $(\varphi)=\varphi\left(F^{\times} \times \operatorname{SL}(2, \mathbb{C})\right)$ is the image of $\varphi$.

- $Z_{L} G^{\circ}\left(\right.$ Image $\varphi$ ) is the centralizer (in ${ }^{L} G^{\circ}$ ) of Image $(\varphi)$.

- $\pi_{0}\left(Z_{L} G^{\circ}(\right.$ Image $\left.\varphi)\right)$ is the finite group whose elements are the connected components of $Z_{L}$ ० (Image $\left.\varphi\right)$.

- $\mathcal{B V}(\varphi)$ is the algebraic variety of all Borel subgroups of ${ }^{L} G^{\circ}$ which contain $\varphi\left(B_{2}\right)$ - where $B_{2}$ is the standard Borel subgroup in $\operatorname{SL}(2, \mathbb{C})$ i.e. $B_{2}$ is the subgroup of $\operatorname{SL}(2, \mathbb{C})$ consisting of all upper triangular matrices in $\operatorname{SL}(2, \mathbb{C})$. A Borel subgroup of ${ }^{L} G^{\circ}$ is a connected solvable algebraic subgroup $B$ of ${ }^{L} G^{\circ}$ which is maximal among the connected solvable algebraic subgroups of ${ }^{L} G^{\circ}$.

- $H^{*}(\mathcal{B V}(\varphi) ; \mathbb{C}$ ) is the cohomology (in the usual sense of algebraic topology), with coefficients $\mathbb{C}$, of the underlying locally compact Hausdorff space of the complex algebraic variety $\mathcal{B} \mathcal{V}(\varphi)$.

- $Z_{L_{G}}$ (Image $\varphi$ ) acts on $\mathcal{B V}(\varphi)$ by conjugation, thus determining a representation (whose vector space is $H^{*}(\mathcal{B V}(\varphi) ; \mathbb{C})$ ) of the finite group $\pi_{0}\left(Z_{L} G^{\circ}(\right.$ Image $\left.\varphi)\right)$. 
A hypothesis in Theorem 2 is that the $p$-adic field $F$ satisfies a mild restriction on its residual characteristic, depending on $G$. Granted this restriction, Theorem 2 states that the ABPS conjecture is valid for any Bernstein component $\operatorname{Irr}(G)_{\mathfrak{s}}$ in the principal series.

Theorem 2. Let $G$ be a connected split reductive p-adic group. Assume that the residual characteristic of the local field $F$ is not a torsion prime for $G$. Let $\operatorname{Irr}(G)_{\mathfrak{s}}$ be a Bernstein component in the principal series of $G$. Then the ABPS conjecture is valid for $\operatorname{Irr}(G)_{\mathfrak{s}}$. In particular, there is a commutative triangle of natural bijections

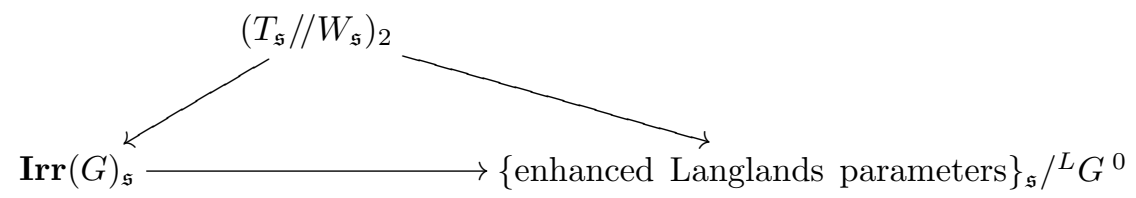

In this triangle \{enhanced Langlands parameters $\}_{\mathfrak{s}} /{ }^{L} G^{0}$ is the set of enhanced Langlands parameters for the Bernstein component $\operatorname{Irr}(G)_{\mathfrak{s}}$ (modulo conjugation by $\left.{ }^{L} G^{0}\right)$.

Theorem 2 extends and generalizes [22] and [30]. Note that [30] assumes that for ramified inducing characters (i.e. all Bernstein components except the Iwahori component) the center of $G$ is connected. In Theorem 2 there is no hypothesis that the center of $G$ is connected.

Brief indication of proof. In the commutative triangle of Theorem 2, the left slanted arrow is defined and is proved bijective by using the representation theory of affine Hecke algebras [33]. The right slanted arrow is defined and is proved bijective by applying the Springer correspondence for affine Weyl groups [21].

Proofs of Theorems 1 and 2 are given in [7].

In $\S 15$ we formulated the ABPS conjecture for quasi-split groups. What happens if $G$ is a connected reductive $p$-adic group which is not quasi-split? Many Bernstein components $\operatorname{Irr}(G)_{\mathfrak{s}}$ have the geometric structure as in the statement in Section 15. However, in some examples there are Bernstein components $\operatorname{Irr}(G)_{\mathfrak{s}}$ which are canonically in bijection not with $\left(T_{\mathfrak{s}} / / W_{\mathfrak{s}}\right)_{2}$ but with $\left(T_{\mathfrak{s}} / / W_{\mathfrak{s}}\right)_{2}$-twisted by a 2-cocyle. See [7] for the definition of $\left(T_{\mathfrak{s}} / / W_{\mathfrak{s}}\right)_{2}$-twisted by a 2 -cocycle. The authors of this note are currently formulating a precise statement of their conjecture for connected reductive $p$-adic groups which are not quasi-split. Our precise statement will be given elsewhere. For groups which are quasi-split but not split, the conjecture is as stated in $\S 15$, i.e. for such groups, there is no change in the conjecture.

\section{Appendix : Geometric Equivalence}

Let $X$ be a complex affine variety and let $k=\mathcal{O}(X)$ be its coordinate algebra. Equivalently, $k$ is a unital algebra over the complex numbers which is commutative, finitely generated, and nilpotent-free. A $k$-algebra is an algebra $A$ over the complex numbers which is a $k$-module (with an evident compatibility between the algebra structure of $A$ and the $k$-module structure of $A$ ). $A$ is of finite type [23] if as a $k$-module $A$ is finitely generated. The $k$-algebra $A$ is not assumed to have a unit 
or to be commutative. This appendix will introduce - for finite type $k$-algebras - a weakening of Morita equivalence called geometric equivalence.

The new equivalence relation preserves the primitive ideal space (i.e. the set of isomorphism classes of simple $A$-modules) and the periodic cyclic homology. However, the new equivalence relation permits a tearing apart of strata in the primitive ideal space which is not allowed by Morita equivalence. The ABPS conjecture asserts that the finite type algebra which Bernstein constructs [12, 13, 31] for any given Bernstein component of a reductive $p$-adic group is geometrically equivalent to the coordinate algebra of the associated extended quotient - and that the geometric equivalence can be chosen so that the resulting bijection between the Bernstein component and the extended quotient has the properties required by the conjecture.

\section{1. k-algebras.}

Let $X$ be a complex affine variety and $k=\mathcal{O}(X)$ its coordinate algebra.

A $k$-algebra is a $\mathbb{C}$-algebra $A$ such that $A$ is a unital (left) $k$-module with:

$$
\lambda(\omega a)=\omega(\lambda a)=(\lambda \omega) a \quad \forall(\lambda, \omega, a) \in \mathbb{C} \times k \times A
$$

and

$$
\omega\left(a_{1} a_{2}\right)=\left(\omega a_{1}\right) a_{2}=a_{1}\left(\omega a_{2}\right) \quad \forall\left(\omega, a_{1}, a_{2}\right) \in k \times A \times A .
$$

Denote the center of $A$ by $Z(A)$.

$$
Z(A):=\{c \in A \mid c a=a c \forall a \in A\}
$$

$k$-algebras are not required to be unital or commutative.

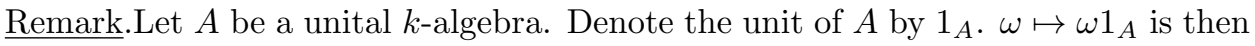
a unital morphism of $\mathbb{C}$-algebras $k \rightarrow Z(A)$. Thus a unital $k$-algebra is a unital $\mathbb{C}$-algebra $A$ with a given unital morphism of $\mathbb{C}$-algebras

$$
k \longrightarrow Z(A) \text {. }
$$

Let $A, B$ be two $k$-algebras. A morphism of $k$-algebras is a morphism of $\mathbb{C}$ algebras

$$
f: A \rightarrow B
$$

which is also a morphism of (left) $k$-modules,

$$
f(\omega a)=\omega f(a) \quad \forall(\omega, a) \in k \times A .
$$

Let $A$ be a $k$-algebra. A representation of $A$ [or a (left) $A$-module] is a $\mathbb{C}$-vector space $V$ with given morphisms of $\mathbb{C}$-algebras

$$
A \longrightarrow \operatorname{Hom}_{\mathbb{C}}(V, V) \quad k \longrightarrow \operatorname{Hom}_{\mathbb{C}}(V, V)
$$

such that

(1) $k \rightarrow \operatorname{Hom}_{\mathbb{C}}(V, V)$ is unital;

(2) $(\omega a) v=\omega(a v)=a(\omega v) \forall(\omega, a, v) \in k \times A \times V$.

Two representations

$$
A \longrightarrow \operatorname{Hom}_{\mathbb{C}}\left(V_{1}, V_{1}\right) \quad k \longrightarrow \operatorname{Hom}_{\mathbb{C}}\left(V_{1}, V_{1}\right)
$$

and

$$
A \longrightarrow \operatorname{Hom}_{\mathbb{C}}\left(V_{2}, V_{2}\right) \quad k \longrightarrow \operatorname{Hom}_{\mathbb{C}}\left(V_{2}, V_{2}\right)
$$

are equivalent (or isomorphic) if there is an isomorphism of $\mathbb{C}$ vector spaces $T: V_{1} \rightarrow$ $V_{2}$ which intertwines the two $A$-actions and the two $k$-actions. 
A representation is irreducible if $A \rightarrow \operatorname{Hom}_{\mathbb{C}}(V, V)$ is not the zero map and there is no sub- $\mathbb{C}$-vector space $W$ of $V$ with:

$$
\{0\} \neq W \quad, \quad W \neq V
$$

and

$$
\omega w \in W \quad \forall(\omega, w) \in k \times W
$$

and

$$
a w \in W \quad \forall(a, w) \in A \times W
$$

$\operatorname{Irr}(A)$ denotes the set of equivalence classes of irreducible representations of $A$.

\subsection{Spectrum preserving morphisms of $k$-algebras.}

Definition. An ideal $I$ in a $k$-algebra $A$ is a $k$-ideal if $\omega a \in I \forall(\omega, a) \in k \times I$. An ideal $I \subset A$ is primitive if there exists an irreducible representation

$$
\varphi: A \rightarrow \operatorname{Hom}_{\mathbb{C}}(V, V) \quad k \longrightarrow \operatorname{Hom}_{\mathbb{C}}(V, V)
$$

with

$$
I=\operatorname{Kernel}(\varphi)
$$

That is,

$$
0 \rightarrow I \hookrightarrow A \stackrel{\varphi}{\rightarrow} \operatorname{Hom}_{\mathbb{C}}(V, V)
$$

is exact.

Remark. Any primitive ideal is a $k$-ideal. $\operatorname{Prim}(A)$ denotes the set of primitive ideals in $A$. The map $\operatorname{Irr}(A) \rightarrow \operatorname{Prim}(A)$ which sends an irreducible representation to its primitive ideal is a bijection if $A$ is a finite type $k$-algebra [23]. Since $k$ is Noetherian, any $k$-ideal in a finite type $k$-algebra $A$ is itself a finite type $k$-algebra.

Definition. Let $A, B$ be two finite type $k$-algebras, and let $f: A \rightarrow B$ be a morphism of $k$-algebras. $f$ is spectrum preserving if

(1) Given any primitive ideal $I \subset B$, there is a unique primitive ideal $L \subset A$ with $L \supset f^{-1}(I)$;

(2) The resulting map

$$
\operatorname{Prim}(B) \rightarrow \operatorname{Prim}(A)
$$

is a bijection.

In this situation the map $\operatorname{Prim}(B) \rightarrow \operatorname{Prim}(A)$ is in fact a homeomorphism [11].

Definition. Let $A, B$ be two finite type $k$-algebras, and let $f: A \rightarrow B$ be a morphism of $k$-algebras. $f$ is spectrum preserving with respect to filtrations if there exist $k$-ideals

$$
0=I_{0} \subset I_{1} \subset \cdots \subset I_{r-1} \subset I_{r}=A \quad \text { in } A
$$

and $k$-ideals

$$
0=L_{0} \subset L_{1} \subset \cdots \subset L_{r-1} \subset L_{r}=B \quad \text { in } B
$$

with $f\left(I_{j}\right) \subset L_{j},(j=1,2, \ldots, r)$ and $I_{j} / I_{j-1} \rightarrow L_{j} / L_{j-1},(j=1,2, \ldots, r)$ is spectrum preserving.

These data determine a bijection $\operatorname{Prim}(B) \rightarrow \operatorname{Prim}(A)$ which, however, need not be continuous. 


\subsection{Algebraic variation of $k$-structure.}

Notation. If $A$ is a $\mathbb{C}$-algebra, $A\left[t, t^{-1}\right]$ is the $\mathbb{C}$-algebra of Laurent polynomials in the indeterminate $t$ with coefficients in $A$.

Definition. Let $A$ be a unital $\mathbb{C}$-algebra, and let

$$
\Psi: k \rightarrow Z\left(A\left[t, t^{-1}\right]\right)
$$

be a unital morphism of $\mathbb{C}$-algebras. Note that $Z\left(A\left[t, t^{-1}\right]\right)=Z(A)\left[t, t^{-1}\right]$. For $\zeta \in \mathbb{C}^{\times}=\mathbb{C}-\{0\}, \operatorname{ev}(\zeta)$ denotes the "evaluation at $\zeta$ " map:

$$
\begin{aligned}
\operatorname{ev}(\zeta): A\left[t, t^{-1}\right] & \rightarrow A \\
\sum a_{j} t^{j} & \mapsto \sum a_{j} \zeta^{j} .
\end{aligned}
$$

Consider the composition

$$
k \stackrel{\Psi}{\longrightarrow} Z\left(A\left[t, t^{-1}\right]\right) \stackrel{\mathrm{ev}(\zeta)}{\longrightarrow} Z(A) .
$$

Denote the unital $k$-algebra so obtained by $A_{\zeta}$. The underlying $\mathbb{C}$-algebra of $A_{\zeta}$ is $A$. Assume that for all $\zeta \in \mathbb{C}^{\times}, A_{\zeta}$ is a finite type $k$-algebra. Then for $\zeta, \zeta^{\prime} \in \mathbb{C}^{\times}, A_{\zeta^{\prime}}$ is obtained from $A_{\zeta}$ by an algebraic variation of $k$-structure.

17.4. Definition and examples. With $k$ fixed, geometric equivalence (for finite type $k$-algebras) is the equivalence relation generated by the two elementary moves:

- Spectrum preserving morphism with respect to filtrations

- Algebraic variation of $k$-structure

Thus two finite type $k$-algebras $A, B$ are geometrically equivalent if there exists a finite sequence $A=A_{0}, A_{1}, \ldots, A_{r}=B$ with each $A_{j}$ a finite type $k$-algebra such that for $j=0,1, \ldots, r-1$ one of the following three possibilities is valid:

(1) $A_{j+1}$ is obtained from $A_{j}$ by an algebraic variation of $k$-structure.

(2) There is a spectrum preserving morphism with respect to filtrations $A_{j} \rightarrow A_{j+1}$.

(3) There is a spectrum preserving morphism with respect to filtrations $A_{j+1} \rightarrow A_{j}$.

To give a geometric equivalence relating $A$ and $B$, the finite sequence of elementary steps (including the filtrations) must be given. Once this has been done, a bijection of the primitive ideal spaces and an isomorphism of periodic cyclic homology [10, 11] are determined:

$$
\operatorname{Prim}(A) \longleftrightarrow \operatorname{Prim}(B) \quad H P_{*}(A) \cong H P_{*}(B)
$$

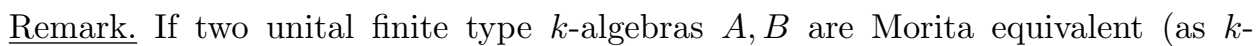
algebras) then they are geometrically equivalent [7]. A Morita equivalence of $A$ and $B$ gives a homeomorphism $\operatorname{Prim}(A) \simeq \operatorname{Prim}(B)$. However, the bijection

$$
\operatorname{Prim}(A) \longleftrightarrow \operatorname{Prim}(B)
$$

obtained from a geometric equivalence might not be a homeomorphism.

Example 1. Let $G$ be a connected reductive complex Lie group with maximal torus T. W denotes the Weyl group

$$
W=N_{G}(T) / T
$$

and $X^{*}(T)$ is the character group of $T . N_{G}(T)$ is the normalizer (in $G$ ) of $T$. The semi-direct product $X^{*}(T) \rtimes W$ is the affine Weyl group of $G$. For each non-zero 
complex number $q$, there is the affine Hecke algebra $\mathcal{H}_{q}(G)$. This is an affine Hecke algebra with equal parameters and $\mathcal{H}_{1}(G)$ is the group algebra of the affine Weyl group:

$$
\mathcal{H}_{1}(G)=\mathbb{C}\left[X^{*}(T) \rtimes W\right]=\mathcal{O}(T) \rtimes W .
$$

Using the action of $W$ on $T$, form the quotient variety $T / W$ and let $k$ be its coordinate algebra,

$$
k=\mathcal{O}(T / W)
$$

For all $q \in \mathbb{C}^{\times}, \mathcal{H}_{q}(G)$ is a unital finite type $k$-algebra. Let $J$ be Lusztig's asymptotic algebra [26, 27, 28].

Except for $q$ in a finite set of roots of unity (none of which is 1) Lusztig constructs a morphism of $k$-algebras

$$
\phi_{q}: \mathcal{H}_{q}(G) \longrightarrow J
$$

which is spectrum preserving with respect to filtrations. The algebra $\mathcal{H}_{q}(G)$ is viewed as a $k$-algebra via the canonical isomorphism

$$
\mathcal{O}(T / W) \cong Z\left(\mathcal{H}_{q}(G)\right) \text {. }
$$

Lusztig's map $\phi_{q}$ maps $Z\left(\mathcal{H}_{q}(G)\right)$ to $Z(J)$ and thus determines a unique $k$-structure for $J$ such that the map $\phi_{q}$ is a morphism of $k$-algebras. $J$ with this $k$-structure will be denoted $J_{q} . \mathcal{H}_{q}(G)$ is then geometrically equivalent to $\mathcal{H}_{1}(G)$ by the three elementary steps

$$
\mathcal{H}_{q}(G) \rightsquigarrow J_{q} \rightsquigarrow J_{1} \rightsquigarrow \mathcal{H}_{1}(G) .
$$

The second elementary step (i.e. passing from $J_{q}$ to $J_{1}$ ) is an algebraic variation of $k$-structure. The first elementary step uses Lusztig's map $\phi_{q}$, and the third elementary step uses Lusztig's map $\phi_{1}$. Hence (provided $q$ is not in the exceptional set of roots of unity - none of which is 1) $\mathcal{H}_{q}(G)$ is geometrically equivalent to $\mathcal{H}_{1}(G)=\mathbb{C}\left[X^{*}(T) \rtimes W\right]=\mathcal{O}(T) \rtimes W$.

As observed in section 12 above, $\operatorname{Irr}\left(\mathcal{H}_{1}(G)\right)=(T / / W)_{2}$. Thus the geometric equivalence of $\mathcal{H}_{q}(G)$ to $\mathcal{H}_{1}(G)$ determines a bijection

$$
(T / / W)_{2} \longleftrightarrow \operatorname{Irr}\left(\mathcal{H}_{q}(G)\right) \text {. }
$$

Example 2. With notation as in example 1, let $\mathcal{H}_{\mathbf{q}}\left(X^{*}(T) \rtimes W\right)$ be the affine Hecke algebra of $X^{*}(T) \rtimes W$ with unequal parameters $\mathbf{q}=\left\{q_{1}, \ldots, q_{k}\right\}$. We assume that $q_{i} \in \mathbb{R}_{>0} . \mathcal{S}_{\mathbf{q}}\left(X^{*}(T) \rtimes W\right)$ denotes the Schwartz completion of $\mathcal{H}_{\mathbf{q}}\left(X^{*}(T) \rtimes W\right)$. In [33] a morphism of Fréchet algebras

$$
\mathcal{S}_{1}\left(X^{*}(T) \rtimes W\right) \rightarrow \mathcal{S}_{\mathbf{q}}\left(X^{*}(T) \rtimes W\right)
$$

is constructed which is spectrum preserving with respect to filtrations.

Example 3. Let $G$ be a connected reductive $p$-adic group, and let $\mathfrak{s} \in \mathfrak{B}(G)$ be any point in the Bernstein spectrum of $G$. $\mathcal{H}_{\mathfrak{s}}$ denotes the unital finite type $\mathcal{O}\left(T_{\mathfrak{s}} / W_{\mathfrak{s}}\right)$ algebra which Bernstein assigns to $\mathfrak{s}$. $\mathcal{H}_{\mathfrak{s}}$ has the property

$$
\operatorname{Irr}\left(\mathcal{H}_{\mathfrak{s}}\right)=\operatorname{Irr}(G)_{\mathfrak{s}}
$$

The ABPS conjecture asserts that the three unital finite type $\mathcal{O}\left(T_{\mathfrak{s}} / W_{\mathfrak{s}}\right)$ algebras

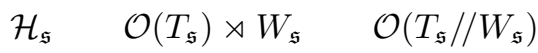

are geometrically equivalent. Results and conjectures of G. Lusztig [26, 27, 28] are relevant to this assertion. According to the ABPS conjecture, the geometric equivalence between $\mathcal{H}_{\mathfrak{s}}$ and $\mathcal{O}\left(T_{\mathfrak{s}}\right) \rtimes W_{\mathfrak{s}}$ is canonical and gives the left slanted 
arrow in the statement of the ABPS conjecture.

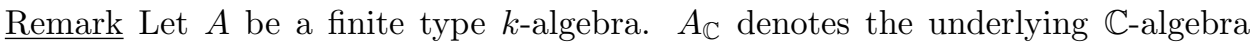
of $A$ - i.e. $A_{\mathbb{C}}$ is obtained from $A$ by forgetting the action of $k$ on $A$. Then an irreducible $A_{\mathbb{C}}$ module in a canonical way becomes an irreducible $A$ module. This gives bijections

$$
\operatorname{Irr}(A) \longleftrightarrow \operatorname{Irr}\left(A_{\mathbb{C}}\right), \quad \operatorname{Prim}(A) \longleftrightarrow \operatorname{Prim}\left(A_{\mathbb{C}}\right) .
$$

\section{REFERENCES}

[1] J. Arthur, A note on L-packets, Pure Appl. Math. Quarterly 2.1 (2006) 199217.

[2] J. Arthur, The endoscopic classification of representations: orthogonal and symplectic groups, Colloquium Publication Series, American Mathematical Society (to appear), available at http://www.claymath.org/cw/arthur/

[3] A.-M. Aubert, P. Baum, R. J. Plymen, The Hecke algebra of a reductive $p$-adic group : a geometric conjecture, in: Non-Commutative Geometry and Number Theory, Aspects of Mathematics 37 (C. Consani and M. Marcolli eds.), 1-34, Vieweg Verlag, Wiesbaden, 2006.

[4] A.-M. Aubert, P. Baum, R. J. Plymen, Geometric structure in the representation theory of $p$-adic groups, C.R. Acad. Sci. Paris, Ser. I, 345, 573-578, 2007.

[5] A.-M. Aubert, P. Baum, R. J. Plymen, Geometric structure in the principal series of the $p$-adic group $G_{2}$, Journal of Representation Theory 15 (2011), $126-169$.

[6] A.-M. Aubert, P. Baum, R. J. Plymen, Geometric structure in the representation theory of reductive $p$-adic groups II, Harmonic Analysis on Reductive p-adic Groups, Contemporary Math. 543, (R. S. Doran, P. J. Sally Jr, L. Spice eds.), 71-90, AMS, Providence, 2011.

[7] A.-M. Aubert, P. Baum, R. J. Plymen, M. Solleveld, Geometric structure and the local Langlands conjecture, preprint, arXiv:1211.0180v2.

[8] A.-M. Aubert, P. Baum, R. J. Plymen, M. Solleveld, The local Langlands correspondence for inner forms of $\mathrm{SL}_{n}$, preprint, arXiv:1305.2638

[9] A.-M. Aubert, P. Baum, R.J. Plymen, M. Solleveld, On the local Langlands correspondence for non-tempered representations, to appear in Münster J. Math.

[10] P. Baum, V. Nistor, Periodic cyclic homology of Iwahori-Hecke algebras. C. R. Acad. Sci. Paris Ser. I Math. 332, 783-788, 2001.

[11] P. Baum, V. Nistor, Periodic cyclic homology of Iwahori-Hecke algebras. $K$ Theory 27 (2002), no. 4, 329-357.

[12] J. N. Bernstein, Le centre de Bernstein, edited by P. Deligne. Travaux en Cours, Representations of reductive groups over a local field, 1-32, Hermann, Paris, 1984.

[13] J. N. Bernstein, K. E. Rumelhart, Representations of p-adic Groups, Harvard Mathematics Department Lecture Notes, 1993.

[14] J. N. Bernstein, S. Gelbart, An introduction to the Langlands program, Birkhäuser, 2003.

[15] A. Borel, Automorphic L-functions, Proc. Symp. Pure Math 33.2 (1979), 2761. 
[16] J. Dixmier, $C^{*}$-algebras, North-Holland, 1977.

[17] M. Harris, R. Taylor, The geometry and cohomology of some simple Shimura varieties, Annals of Math. Studies 151, Princeton University Press, Princeton NJ, 2001. Springer-Verlag, New York NJ, 1977

[18] G. Henniart, Une preuve simple de conjectures de Langlands pour GL $(n)$ sur un corps $p$-adique, Inv. Math. 139 (2000), 439-455.

[19] K. Hiraga, H. Saito, On L-packets for inner forms of $\mathrm{SL}_{n}$, Mem. Amer. Math. Soc. 215 (2012).

[20] K. Iwasawa, Local class field theory, Oxford math. monograph, 1986.

[21] S.-I. Kato, A realization of irreducible representations of affine Weyl groups, Indag. Math. 45.2 (1983), 193-201.

[22] D. Kazhdan, G. Lusztig, Proof of the Deligne-Langlands conjecture for Hecke algebras, Invent. Math. 87 (1987) 153-215.

[23] D. Kazhdan, V. Nistor, P. Schneider, Hochschild and cyclic homology of finite type algebras, Sel. Math. New Ser. 4.2 (1998), 321-359

[24] R. Langlands, Problems in the theory of automorphic forms, pp. 18-61. in: Lectures in Modern Analysis and Applications, Lecture Notes in Math. 170 (1970), Springer, New-York.

[25] G. Lusztig, Some examples of square integrable representations, Trans. AMS 277 (1983), 623-653.

[26] G. Lusztig, Cells in affine Weyl groups III, J. Fac. Sci. Univ. Tokyo, Sect IA, Math. 34 (1987), 223-243.

[27] G. Lusztig, Cells in affine Weyl groups IV, J. Fac. Sci. Univ. Tokyo, Sect IA, Math. 36 (1989), 297-328.

[28] G. Lusztig, Representations of affine Hecke algebras,Astérisque 171-172 (1989), 73-84.

[29] C.P. Mok, Endoscopic classification of representations of quasi-split unitary groups, arXiv:1206.0882, Memoirs of the AMS, to appear.

[30] M. Reeder, Isogenies of Hecke algebras and a Langlands correspondence for ramified principal series representations, Representation Theory 6 (2002), 101126.

[31] D. Renard, Représentations des groupes réductifs p-adiques, Cours Spécialisés 17, Société Mathématique de France, 2010. Annals of Mathematics Studies 123, Princeton University Press, 1990

[32] P. Scholze, The local Langlands correspondence for $\mathrm{GL}_{n}$ over $p$-adic fields. Invent. Math. 192 (2013) 663 - 715.

[33] M. Solleveld, On the classification of irreducible representations of affine Hecke algebras with unequal parameters, Representation Theory 16 (2012), 1-87.

[34] D. Vogan, The local Langlands conjecture, pp. 305-379 in: Representation theory of groups and algebras, Contemp. Math. 145, Amer. Math. Soc., 1993.

[35] A.V. Zelevinsky, Induced representations of reductive $p$-adic groups II. On irreducible representations of GL(n), Ann. Sci. École Norm. Sup. (4) 13.2 (1980), $165-210$. 
Institut de Mathématiques de Jussieu - Paris Rive Gauche, U.M.R. 7586 du C.N.R.S., U.P.M.C., 4 Place Jussieu 75005 Paris, France

E-mail address: aubert@math.jussieu.fr

Mathematics Department, Pennsylvania State University, University Park, PA 16802, USA

E-mail address: baum@math.psu.edu

School of Mathematics, Southampton University, Southampton SO17 1BJ, England and School of Mathematics, Manchester University, Manchester M13 9PL, England

E-mail address: r.j.plymen@soton.ac.uk plymen@manchester.ac.uk

Radboud Universiteit Nijmegen, Heyendaalseweg 135, 6525AJ Nijmegen, the NetherLANDS

E-mail address: m.solleveld@science.ru.nl 